\title{
HD 80606: searching for the chemical signature of planet formation $\star, \star \star, \star \star \star$
}

\author{
C. Saffe ${ }^{1,2}$, M. Flores ${ }^{1}$, and A. Buccino ${ }^{3,4}$ \\ ${ }^{1}$ Instituto de Ciencias Astronómicas, de la Tierra y del Espacio (ICATE-CONICET), C.C 467, 5400 San Juan, Argentina \\ e-mail: csaffe,mflores@icate-conicet.gob.ar \\ 2 Universidad Nacional de San Juan (UNSJ), Facultad de Ciencias Exactas, Físicas y Naturales (FCEFN), San Juan, Argentina \\ 3 Instituto de Astronomía y Física del Espacio (IAFE-CONICET), Buenos Aires, Argentina \\ e-mail: abuccino@iafe.uba.ar \\ ${ }^{4}$ Departamento de Física, Facultad de Ciencias Exactas y Naturales (FCEN), Universidad de Buenos Aires (UBA), Buenos Aires, \\ Argentina
}

Received 1 June 2015 / Accepted 28 July 2015

\begin{abstract}
Context. Binary systems with similar components are ideal laboratories that allow several physical processes to be tested, such as the possible chemical pattern imprinted by the planet formation process.

Aims. We explore the probable chemical signature of planet formation in the remarkable binary system HD 80606-HD 80607. The star HD 80606 hosts a giant planet with $\sim 4 M_{\text {Jup }}$ detected by both transit and radial velocity techniques, which is one of the most eccentric planets detected to date. We study condensation temperature $T_{\mathrm{c}}$ trends of volatile and refractory element abundances to determine whether there is a depletion of refractories, which could be related to the terrestrial planet formation.

Methods. We carried out a high-precision abundance determination in both components of the binary system via a line-by-line, strictly differential approach. First, we used the Sun as a reference and then we used HD 80606. The stellar parameters $T_{\text {eff }}, \log g$, [Fe/H] and $v_{\text {turb }}$ were determined by imposing differential ionization and excitation equilibrium of Fe I and Fe II lines, with an updated version of the program FUNDPAR, together with plane-parallel local thermodynamic equilibrium (LTE) ATLAS9 model atmospheres and the MOOG code. Then, we derived detailed abundances of 24 different species with equivalent widths and spectral synthesis with the program MOOG. The chemical patterns were compared with the solar-twins $T_{\mathrm{c}}$ trends of Meléndez et al. (2009, AJ, 704, L66) and with a sample of solar-analogue stars with $[\mathrm{Fe} / \mathrm{H}] \sim+0.2$ dex from Neves et al. (2009, A\&A, 497, 563). The $T_{\mathrm{c}}$ trends were also compared mutually between both stars of the binary system.

Results. From the study of $T_{\mathrm{c}}$ trends, we concluded that the stars HD 80606 and HD 80607 do not seem to be depleted in refractory elements, which is different for the case of the Sun. Then, following the interpretation of Meléndez et al. (2009), the terrestrial planet formation would have been less efficient in the components of this binary system than in the Sun. The lack of a trend in refractory elements with $T_{\mathrm{c}}$ between both stars implies that the presence of a giant planet do not neccesarily imprint a chemical signature in their host stars, similar to the recent result of Liu et al. (2014, MNRAS, 442, L51). This is also in agreement with Meléndez et al. (2009), who suggest that the presence of close-in giant planets might prevent the formation of terrestrial planets. Finally, we speculate about a possible, ejected or non-detected, planet around the star HD 80607.
\end{abstract}

Key words. stars: abundances - planetary systems - binaries: general - stars: individual: HD 80606

\section{Introduction}

Main-sequence stars with giant planets are, on average, metalrich compared to stars without planetary mass companions (e.g. Santos et al. 2004, 2005; Fischer \& Valenti 2005). On the other hand, Neptune-like or super-Earth planets do not seem to be preferentially formed around metal-rich stars (e.g. Udry et al. 2006; Sousa et al. 2008). Meléndez et al. (2009, hereafter M09) have further suggested that small chemical anomalies, rather

^ The data presented herein were obtained at the W.M. Keck Observatory, which is operated as a scientific partnership among the California Institute of Technology, the University of California, and the National Aeronautics and Space Administration. The Observatory was made possible by the generous financial support of the W.M. Keck Foundation.

$\star \star$ Table 1 is available in electronic form at http: //wwW . aanda . org $\star \star \star$ The reduced spectra (FITS files) are only available at the CDS via anonymous ftp to cdsarc.u-strasbg. fr $(130.79 .128 .5)$ or via http: //cdsarc.u-strasbg.fr/viz-bin/qcat?]/A+A/582/A17 than a global excess of metallicity, are a possible signature of terrestrial planet formation. The authors showed that the Sun is deficient in refractory elements relative to volatile when compared to solar twins, suggesting that the refractory elements depleted in the solar photosphere are possibly locked up in terrestrial planets and/or in the cores of giant planets.

Most binary stars are believed to have formed from a common molecular cloud. This is supported both by observations of binaries in star-forming regions (e.g. Reipurth et al. 2007; Vogt et al. 2012; King et al. 2012) and by numerical models of binary formation (e.g. Reipurth \& Mikkola 2012; Kratter 2011). These systems are ideal laboratories to look for possible chemical differences between their components, especially for physically similar stars that help to minimize the errors. For the case of main-sequence stars, Desidera et al. (2004) studied the components of 23 wide binary stars and showed that most pairs present almost identical abundances, with only four pairs showing differences between 0.02 dex and 0.07 dex. A similar conclusion was reached by Desidera et al. (2006), showing that only 6 out 
of 33 southern binary stars with similar components present differences between 0.05 and 0.09 dex. The origin of the slight differences in these few cases is not totally clear, and a possible explanation lies the planet formation process (e.g. Gratton et al. 2001; Desidera et al. 2004, 2006).

There have been very few detailed studies of binary systems that have similar components, in which one of the stars hosts a planet. For instance, the binary system $16 \mathrm{Cyg}$ is composed of a pair of stars with spectral types G1 V + G2 V, and the B component hosts a giant planet of $\sim 1.5 M_{\text {Jup }}$ (Cochran et al. 1997). This system has received the attention of many different works on chemical abundances in stars. Takeda (2005) and Schuler et al. (2011) suggested that both stars present the same chemical composition, while other studies found that $16 \mathrm{Cyg}$ A is more metalrich than the B component (Laws \& Gonzalez 2001; Ramírez et al. 2011; Tucci Maia et al. 2014). In particular, Tucci Maia et al. (2014) also find a trend between refractories and the condensation temperature $T_{\mathrm{c}}$, which could be interpreted as a signature of the rocky accretion core of the giant planet $16 \mathrm{Cyg} \mathrm{Bb}$. Another example is the binary system HAT-P-1, composed of an F8 V + G0 V pair, in which the cooler star hosts a $\sim 0.53 M_{\text {Jup }}$ transiting planet (Bakos et al. 2007). Recently, Liu et al. (2014) found almost the same chemical abundances in both stars and concluded that the presence of giant planets does not necessarily imply differences in their composition. Both members of the binary system present an identical positive correlation with $T_{\mathrm{c}}$, suggesting that the terrestrial formation process was probably less efficient in this system. Liu et al. (2014) also discuss why the chemical signature of planet formation is detected in the binary system $16 \mathrm{Cyg}$, but it is not detected in the HAT-P-1 system. The planet $16 \mathrm{Cyg} \mathrm{Bb}\left(\sim 1.5 M_{\text {Jup }}\right)$ is more massive than the planet HAT-P-1 $\mathrm{Bb}\left(\sim 0.5 M_{\text {Jup }}\right)$, which facilitates the imprint of the chemical signature in their host stars. The stellar masses in the binary system HAT-P-1 (1.16 and $1.12 M_{\odot}$, Bakos et al. 2007) are slightly higher than in the system 16 Cyg (1.05 and $1.00 M_{\odot}$, Ramírez et al. 2011). This implies less massive convection zones in the stars of the system HAT-P-1, i.e. more prone to imprint the chemical signature, and shorter pre-main-sequence disc lifetimes, i.e. more difficult to imprint the chemical signature. These points illustrate how complicated and challenging it could be to determine the possible effects of planet formation using stellar abundances. Then, there is a need for additional stars hosting planets in binary systems to be compared through a highprecision abundance determination.

Naef et al. (2001) first detected a giant planet around the solar-type star HD 80606 using radial-velocity measurements, which is the primary of the wide binary system HD 80606-HD 80607 (components A and B). To date, there is no planet detected around the B component. The separation between A and B stars is 21.1" (e.g. Dommanget \& Nys 2002), corresponding to $\sim 1000 \mathrm{AU}$ at the distance of about $60 \mathrm{pc}$ (Laughlin et al. 2009). This binary system is particularly notable for several reasons. Both stars present very similar fundamental parameters (their effective temperatures only differ by $67 \mathrm{~K}$ and their superficial gravities by $0.01 \mathrm{dex}$, as we see later). The reported spectral types are G5 V + G5 V, as described in the Hipparcos catalogue. This makes this system a new member of the selected group of binaries with very similar components. The exoplanet HD $80606 \mathrm{~b}$ have a period of 111.8 days and one of the most eccentric orbits to date $(e=0.927$, Naef et al. 2001), probably because of the influence of the B star (Wu \& Murray 2003). Besides the radial-velocity detection, Laughlin et al. (2009) reported a secondary transit for HD $80606 \mathrm{~b}$ using $8 \mu \mathrm{m}$ Spitzer observations, while Moutou et al. (2009) detected the primary transit of the planet and measured a planet radius of $0.9 M_{\mathrm{Jup}}$. Then, future observations of the atmosphere of this transiting planet could be compared to the natal chemical environment established by a binary star elemental abundances, as suggested by Teske et al. (2013). These significant features motivated this study, exploring the possible chemical signature of planet formation in this remarkable system.

There are some previous abundance measurements of HD 80606 in the literature. A number of elements show noticeable discrepancies in the reported values. Notably, using the same stellar parameters, the $\mathrm{Na}$ abundance was reported as $+0.30 \pm 0.05$ dex and $+0.53 \pm 0.12$ dex (Beirao et al. 2005; Mortier et al. 2013), while the $\mathrm{Si}$ abundance resulted in $+0.40 \pm 0.09$ dex and $+0.27 \pm 0.06 \mathrm{dex}$ (Mortier et al. 2013; Gilli et al. 2006). These differences also encouraged this work. We perform a high-precision abundance study analysing both members of this unique binary system with a line-by-line differential approach, aiming to detect a slight contrast between their components.

This work is organized as follows. In Sect. 2 we describe the observations and data reduction, while in Sect. 3 we present the stellar parameters and chemical abundance analysis. In Sect. 4 we show the results and discussion, and finally in Sect. 5 we highlight our main conclusions.

\section{Observations and data reduction}

Stellar spectra of HD 80606 and HD 80607 were obtained with the High Resolution Echelle Spectrometer (HIRES) attached on the right Nasmyth platform of the Keck 10-m telescope on Mauna Kea, Hawaii. The slit used was B2 with a width of 0.574 arcsec, which provides a measured resolution of $\sim 67000$ at $\sim 5200 \AA^{1}$. The spectra were downloaded from the Keck Observatory Archive $(\mathrm{KOA})^{2}$, under the program ID A271Hr.

The observations were taken on March, 15th 2011 with HD 80607 observed immediately after HD 80606, using the same spectrograph configuration. The exposure times were $3 \times$ $300 \mathrm{~s}$ for both targets. We measured a signal-to-noise ratio $S / N \sim 330$ for each of the binary components. The asteroid Iris was also observed with the same spectrograph set-up achieving a similar $\mathrm{S} / \mathrm{N}$, to acquire the solar spectrum useful for reference in our (initial) differential analysis. We note however that the final differential study with the highest abundance precision is between HD 80606 and HD 80607 because of their high degree of similarity.

Our resolving power is approximately 40 percent higher than those reported in previous works (Ecuvillon et al. 2006; Gilli et al. 2006; Mortier et al. 2013). Even for a similar resolution and $\mathrm{S} / \mathrm{N}$, however, the differential line-by-line approach applied here results in a significant improvement in the derived abundances, as we show in the follwoing sections.

We reduced the HIRES spectra using the data reduction package $\mathrm{MAKEE}^{3}$ (MAuna Kea Echelle Extraction), which performs the usual reduction process including bias subtraction, flat fielding, spectral order extractions, and wavelength calibration. The continuum normalization and other operations (Doppler

\footnotetext{
http://www2 . keck.hawaii . edu/inst/hires/slitres.html

http://www2 . keck . hawaii . edu/koa/koa.html

http://www.astro. caltech.edu/ tb/makee/
} 
correction and combining spectra) was perfomed using Image Reduction and Analysis Facility (IRAF) ${ }^{4}$.

\section{Stellar parameters and chemical abundance analysis}

We started by measuring the equivalent widths (EW) of Fe I and Fe II lines in the spectra of our program stars using the IRAF task splot, and then continued with other chemical species. The lines list and relevant laboratory data (such as excitation potential and oscilator strengths) were taken from Liu et al. (2014), Meléndez et al. (2014), and then extended with data from Bedell et al. (2014), who carefully selected lines for a high-precision abundance determination. This data, including the measured EWs, are presented in Table 1.

The fundamental parameters $\left(T_{\text {eff }}, \log g,[\mathrm{Fe} / \mathrm{H}], v_{\text {turb }}\right)$ of HD 80606 and HD 80607 were derived by imposing excitation and ionization balance of Fe I and Fe II lines. We used an updated version of the program FUNDPAR (Saffe 2011), which uses the MOOG code (Sneden 1973) together with ATLAS9 model atmospheres (Kurucz 1993) to search for the appropriate solution. The procedure uses explicity calculated (i.e. non-interpolated) plane-parallel local thermodynamic equilibrium (LTE) Kurucz's model atmospheres with ATLAS9 and NEWODF opacities (Castelli \& Kurucz 2003).

We tested the model atmospheres using the PERL ${ }^{5}$ program ifconv.pl, which is available in the $\mathrm{web}^{6}$ together with the Linux port of the Kurucz's programs. The code checks both the convergence of the stellar flux and the flux derivative in the ATLAS9 models, at different Rosseland optical depths. The convergence could be a problem in the outermost layers of models calculated with very low $T_{\text {eff }}(\sim 3500 \mathrm{~K}$ or less) and very low $\log g$, as reported on the same page. Under these conditions, even the LTE hypothesis probably does not hold, however, the Kurucz's models used here are far from these values and have been tested using the mentioned program.

The relative spectroscopic equilibrium was achieved using differential abundances $\delta_{i}$ for each line $i$, defined as

$\delta_{i}=A_{i}^{*}-A_{i}^{\mathrm{ref}}$

where $A_{i}^{*}$ and $A_{i}^{\text {ref }}$ are the abundances in the star of interest and in the reference $\operatorname{star}^{7}$. The same equilibrium conditions used in Saffe (2011) are written for the differential case as the following:

$$
\begin{aligned}
& s_{1}=\frac{\partial\left(\delta_{i}^{\mathrm{FeI}}\right)}{\partial\left(\chi^{\mathrm{exc}}\right)}=0, \\
& s_{2}=\frac{\partial\left(\delta_{i}^{\mathrm{FeI}}\right)}{\partial\left(E W_{r}\right)}=0, \\
& D=\left\langle\delta_{i}^{\mathrm{FeI}}\right\rangle-\left\langle\delta_{i}^{\mathrm{FeII}}\right\rangle=0, \\
& \left\langle\delta_{i}^{\mathrm{FeI}}\right\rangle_{(\mathrm{INP})}-\left\langle\delta_{i}^{\mathrm{FeI}}\right\rangle_{(\mathrm{OUT})}=0,
\end{aligned}
$$

\footnotetext{
4 IRAF is distributed by the National Optical Astronomical Observatories, which is operated by the Association of Universities for Research in Astronomy, Inc. under a cooperative agreement with the National Science Foundation.

5 PERL (Practical Extraction and Reporting Language) is a high-level interpreted programming language.

6 http://atmos.obspm.fr/index.php/documentation/7

7 We use the usual abundance definition $A(X)=\log \left(N_{\mathrm{X}} / N_{\mathrm{H}}\right)+12$.
}

where $\chi^{\text {exc }}$ is the excitation potential and $\mathrm{EW}_{r}$ is the logarithm of the reduced equivalent width. The symbol " \langle\rangle " denotes the abundance average of the different lines, while (INP) and (OUT) correspond to the input and output abundances in the program MOOG. The values $s_{1}$ and $s_{2}$ are the slopes in the plots of abundance vs. $\chi^{\mathrm{exc}}$ and abundance vs. $\mathrm{EW}_{r}$. With this approach, Eqs. (2) and (3) show the independence of differential abundances with the excitation potential and equivalent widths (by requiring null slopes $s_{1}$ and $s_{2}$ ), and Eq. (4) is the differential equilibrium between Fe I and Fe II abundances. Equation (5) expresses the imposed condition to the input and output abundances in the final solution. The updated version of the program FUNDPAR searches for a solution that simultaneously verifies the conditions 2 to 5 . The use of the four mentioned conditions ( 2 to 5) were previously tested (for the "classical" non-differential case) using 61 main-sequence stars (Saffe 2011), 223 giant stars (Jofré et al. 2015), and nine early-type stars (Saffe \& Levato 2014), obtaining very similar parameters to the literature. Then, we applied these conditions for the differential line-by-line case, deriving for both stars stellar parameters in agreement with the literature and with lower errors, as we see later.

Stellar parameters of HD 80606 and HD 80607 were differentially determined using the Sun as standard in an initial approach, and then we recalculate the parameters of HD 80607 using HD 80606 as reference. First, we determined absolute abundances for the Sun using $5777 \mathrm{~K}$ for $T_{\text {eff }}, 4.44 \operatorname{dex}$ for $\log g$ and an initial $v_{\text {turb }}$ of $1.0 \mathrm{~km} \mathrm{~s}^{-1}$. Then, we estimated $v_{\text {turb }}$ for the Sun with the usual method of requiring zero slope in the absolute abundances of Fe I lines versus $\mathrm{EW}_{r}$ and obtained a final $v_{\text {turb }}$ of $0.91 \mathrm{~km} \mathrm{~s}^{-1}$. We note however that the exact values are not crucial for our strictly differential study (see e.g. Bedell et al. 2014).

The next step was the determination of stellar parameters of HD 80606 and HD 80607 using the Sun as standard. For HD 80606, the resulting stellar parameters were $T_{\text {eff }}=5573 \pm$ $43 \mathrm{~K}, \log g=4.32 \pm 0.14 \mathrm{dex},[\mathrm{Fe} / \mathrm{H}]=0.330 \pm 0.005 \mathrm{dex}$, and $v_{\text {turb }}=0.89 \pm 0.09 \mathrm{~km} \mathrm{~s}^{-1}$. For HD 80607, we obtained $T_{\text {eff }}=$ $5506 \pm 21 \mathrm{~K}, \log g=4.31 \pm 0.11 \mathrm{dex},[\mathrm{Fe} / \mathrm{H}]=0.316 \pm 0.006 \mathrm{dex}$, and $v_{\text {turb }}=0.86 \pm 0.17 \mathrm{~km} \mathrm{~s}^{-1}$. The metallicity of the A star is slightly higher than B by 0.014 dex. Figures 1 and 2 shows the plots of abundance vs. excitation potential and abundance vs. $\mathrm{EW}_{r}$ for both stars. Filled and empty points correspond to Fe I and Fe II, while the dashed lines are linear fits to the differential abundance values.

The errors in the stellar parameters were derived as follows. We estimated the change in the "observable" quantities (i.e. the slopes $s_{1}$ and $s_{2}$ and the abundance differences shown in Eqs. (4) and (5)), corresponding to individual changes in the measured parameters $T_{\text {eff }}, \log g,[\mathrm{Fe} / \mathrm{H}]$, and $v_{\text {turb }}(50 \mathrm{~K}, 0.05 \mathrm{dex}, 0.05 \mathrm{dex}$, $0.05 \mathrm{~km} \mathrm{~s}^{-1}$ ). The mentioned changes in the observables are easily read in a normal execution of FUNDPAR. A similar procedure was used previously to calculate these changes (see e.g. Table 2 of Epstein et al. 2010). The differences are then used to estimate the standard deviation terms, which correspond to independent parameters in the usual error propagation. For instance, the mentioned variation of 0.05 dex in $\log g$ for HD 80606 produce a variation in $\mathrm{D}$ (the abundance difference between Fe I and Fe II defined in Eq. (4)) of $\sim 0.028$ dex. Then, the individual error term in $\log g$, which only corresponds to the variation with $\mathrm{D}$, is estimated in a first-order approximation as $(0.05 / 0.028)^{2} \sigma_{\mathrm{D}}^{2}$, where $\sigma_{\mathrm{D}}$ is the standard deviation of the $\mathrm{D}$ values. The $\mathrm{D}$ values are estimated using different Fe lines as $\sigma_{\mathrm{D}}^{2} \simeq \sigma_{\mathrm{FeI}}^{2}+\sigma_{\mathrm{FeII}}^{2}$. Then, we also take the covariance terms into account using the 

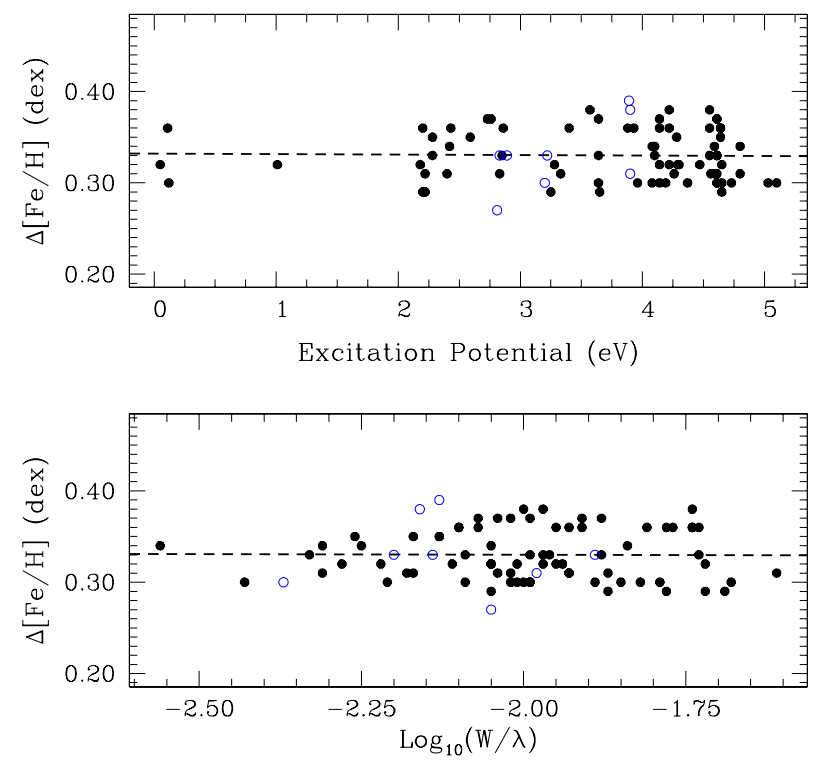

Fig. 1. Differential abundance vs. excitation potential (upper panel) and differential abundance vs. reduced EW (lower panel), for HD 80606 relative to the Sun. Filled and empty points correspond to Fe I and Fe II, respectively. The dashed line is a linear fit to the abundance values.
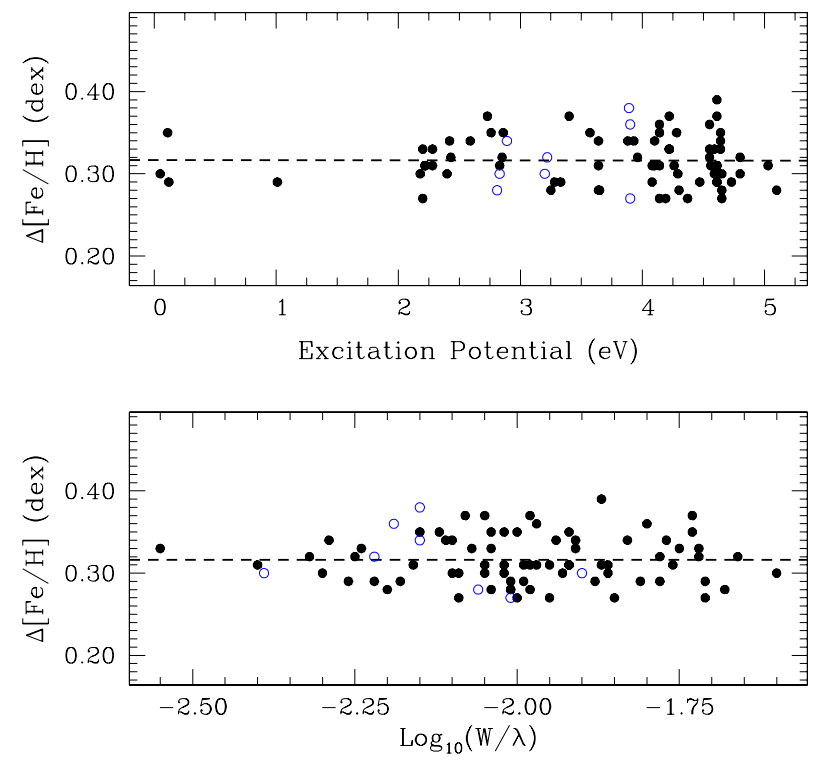

Fig. 2. Differential abundance vs. excitation potential (upper panel) and differential abundance vs. reduced EW (lower panel), for HD 80607 relative to the Sun. Filled and empty points correspond to $\mathrm{Fe}$ I and Fe II, respectively. The dashed line is a linear fit to the abundance values.

Cauchy-Schwarz inequality ${ }^{8}$, which allows us to calculate the mutual covariances with the (previously calculated) individual standard deviations. With this approach, the inequality ensures that our final error adopted is not underestimated.

We repeated the process with HD 80606 as the reference star instead of the Sun, fixing the parameters of the A component to perform the differential analysis. Figure 3 shows the plots of abundance vs. excitation potential and abundance vs. $\mathrm{EW}_{r}$, using similar symbols to those used in Figs. 1 and 2. A visual inspection of Figs. 3 and 1 shows the lower dispersion in the HD 80607 differential abundance values using HD 80606 as a reference

\footnotetext{
8 The inequality for two variables $\mathrm{x}$ and $\mathrm{y}$ is $\sigma_{x y}^{2}<=\sigma_{x}^{2} \sigma_{y}^{2}$, where $\sigma_{x y}^{2}$ is the mutual covariance term and $\sigma_{x}, \sigma_{y}$ are the individual dispersions.
}
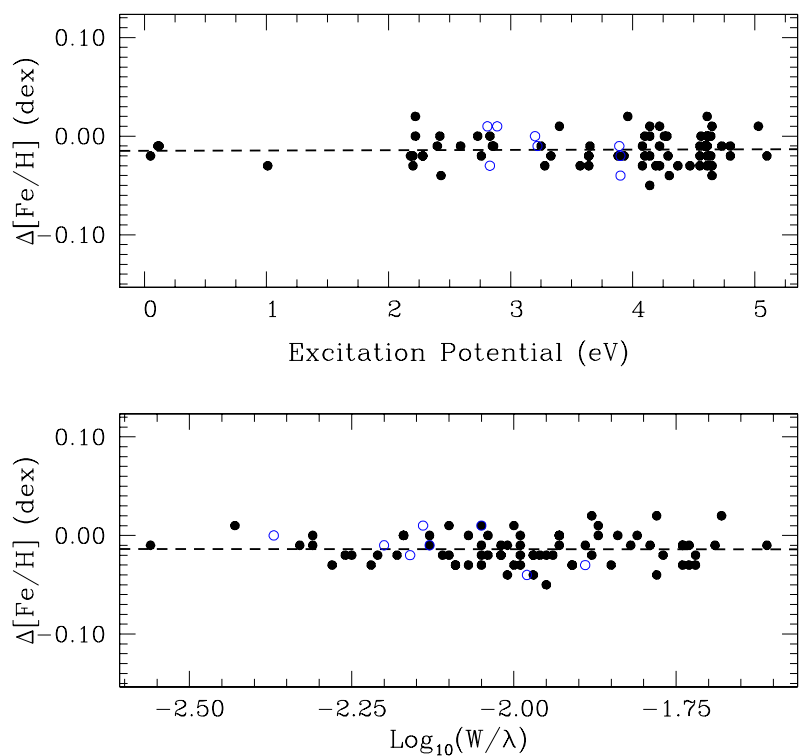

Fig. 3. Differential abundance vs. excitation potential (upper panel) and differential abundance vs. reduced EW (lower panel), for HD 80607 relative to HD 80606. Filled and empty points correspond to Fe I and Fe II, respectively. The dashed line is a linear fit to the data.

star. The resulting stellar parameters for HD 80607 yielded the same results as when we used the Sun as a reference, but with lower dispersions: $T_{\text {eff }}=5506 \pm 14 \mathrm{~K}, \log g=4.31 \pm 0.08 \mathrm{dex}$, $[\mathrm{Fe} / \mathrm{H}]=-0.014 \pm 0.003 \mathrm{dex}$ and $v_{\text {turb }}=0.86 \pm 0.07 \mathrm{~km} \mathrm{~s}^{-1}$. Then, we found that the metallicity results for HD 80607 was slightly lower than the results for HD 80606 by 0.014 dex, equal to the value found using the Sun as reference.

The stellar parameters derived for the A and B stars are similar to those previously determined in the literature. Gonzalez \& Laws (2007) derived $[\mathrm{Fe} / \mathrm{H}]=0.349 \pm 0.073 \mathrm{dex}$ for HD 80606, while Santos et al. (2004) derived $\left(T_{\mathrm{eff}}, \log g\right.$, $\left.[\mathrm{Fe} / \mathrm{H}], v_{\text {turb }}\right)=(5574 \pm 72 \mathrm{~K}, 4.46 \pm 0.20 \mathrm{dex}, 0.32 \pm 0.09 \mathrm{dex}$, $1.14 \pm 0.09 \mathrm{~km} \mathrm{~s}^{-1}$ ) for HD 80606, i.e. only $1 \mathrm{~K}$ difference compared to our result and 0.01 dex difference in $[\mathrm{Fe} / \mathrm{H}]$. The $\log g$ and $v_{\text {turb }}$ values differ by 0.14 dex and $0.25 \mathrm{~km} \mathrm{~s}^{-1}$, respectively. The stellar parameters derived by Santos et al. were then adopted in other works (Ecuvillon et al. 2006; Gilli et al. 2006; Mortier et al. 2013). For HD 80607, Koleva \& Vazdekis (2012) derived $T_{\text {eff }}=5389 \pm 45 \mathrm{~K}, \log g=3.99 \pm 0.18 \mathrm{dex}$, and $[\mathrm{Fe} / \mathrm{H}]=$ $+0.35 \pm 0.06$ dex, but adopting a fixed $v_{\text {turb }}=2.0 \mathrm{~km} \mathrm{~s}^{-1}$ for all the stars in their sample.

Once the stellar parameters of the binary components were determined using iron lines, we computed abundances for all remaining elements: C I, O I, Na I, Mg I, Al I, Si I, S I, Ca I, Sc I, Sc II Ti I, Ti II, V I, Cr I, Cr II, Mn I, Fe I, Fe II, Co I, $\mathrm{Ni}$ I, Cu I, Sr I, Y II, and Ba II. The hyperfine structure splitting (HFS) was considered for V I, Mn I, Co I, Cu I, and Ba II, using the HFS constants of Kurucz \& Bell (1995) and performing spectral synthesis for these species. In Fig. 4 we show an example of the observed and synthethic spectra in the region of the line Ba II 5853.67 $\AA$ for the star HD 80606. The same spectral lines were measured in both stars. We applied non-local thermodynamic equilibrium (NLTE) corrections to the O I triplet following Ramírez et al. (2007) instead of Fabbian et al. (2009) or Takeda (2003) because those works do not include corrections for $[\mathrm{Fe} / \mathrm{H}]>0$. The abundances for O I (NLTE) are $\sim 0.11 \mathrm{dex}$ lower than LTE values, adopting the same correction within errors for both stars given very similar stellar parameters. We 


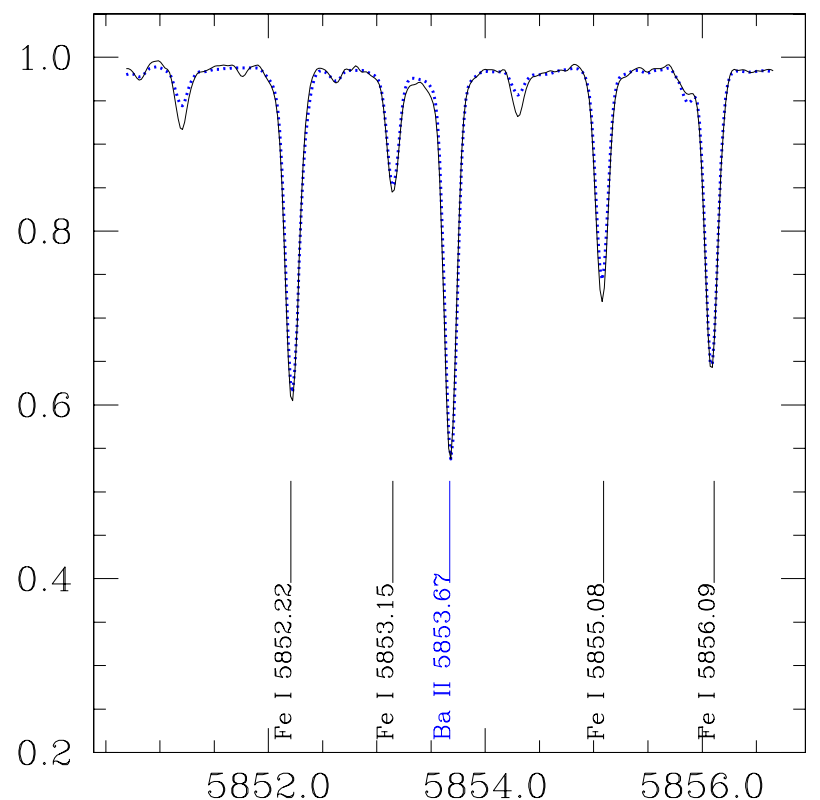

Fig. 4. Observed and synthethic spectra (continuous and dotted lines) near the line Ba II 5853.67 $\AA$ for HD 80606. Some line identifications are shown.

also applied NLTE corrections to Ba II following Korotin et al. (2011), who clearly show that NLTE abundances are higher than LTE values for $[\mathrm{Fe} / \mathrm{H}]>0$.

In Table 2 we present the final differential abundances $[\mathrm{X} / \mathrm{Fe}]^{9}$ of HD 80606 and HD 80607 relative to the Sun, and the differential abundances of HD 80607 using HD 80606 as the reference star. We present both the observational errors $\sigma_{\text {obs }}$ (estimated as $\sigma / \sqrt{(n-1)}$, where $\sigma$ is the standard deviation of the different lines) and systematic errors due to uncertainties in the stellar parameters $\sigma_{\text {par }}$ (by adding quadratically the abundance variation when modifying the stellar parameters by their uncertainties), as well as the total error $\sigma_{\text {TOT }}$ obtained by quadratically adding $\sigma_{\mathrm{obs}}, \sigma_{\mathrm{par}}$ and the error in $[\mathrm{Fe} / \mathrm{H}]$.

\section{Results and discussion}

We present, in Figs. 5 and 6, the differential abundances of HD 80606 and HD 80607 relative to the Sun. We took the condensation temperatures from the $50 \% T_{\mathrm{c}}$ values derived by Lodders (2003). The individual comparison between one component (e.g. HD 80606) and the Sun, is possibly affected by Galactic chemical evolution (GCE) effects because of their different chemical natal environments (see e.g. Tayouchi \& Chiba 2014; Mollá et al. 2015, and references therein). On the other hand, if we assume that the stars of the binary system were born at the same place and time, we discard the GCE effects when comparing the components between them differentially; this is an important advantage of this method. Then, we corrected with GCE effects (only when comparing star-Sun) by adopting the fitting trends of González Hernández et al. (2013); see their Fig. 2, the plots of $[\mathrm{X} / \mathrm{Fe}]$ vs. $[\mathrm{Fe} / \mathrm{H}])$ to derive the values of $[\mathrm{X} / \mathrm{Fe}]$ at $[\mathrm{Fe} / \mathrm{H}] \sim 0.32$ dex. A similar procedure was previously used by Liu et al. (2014) to correct the abundances in the binary system HAT-P-1 with GCE. Filled points in Figs. 5 and 6 correspond to the differential abundances for the stars HD 80606 and HD 80607, respectively. For reference, we also included in these

\footnotetext{
9 We used the standard notation $[\mathrm{X} / \mathrm{Fe}]=[\mathrm{X} / \mathrm{H}]-[\mathrm{Fe} / \mathrm{H}]$.
}

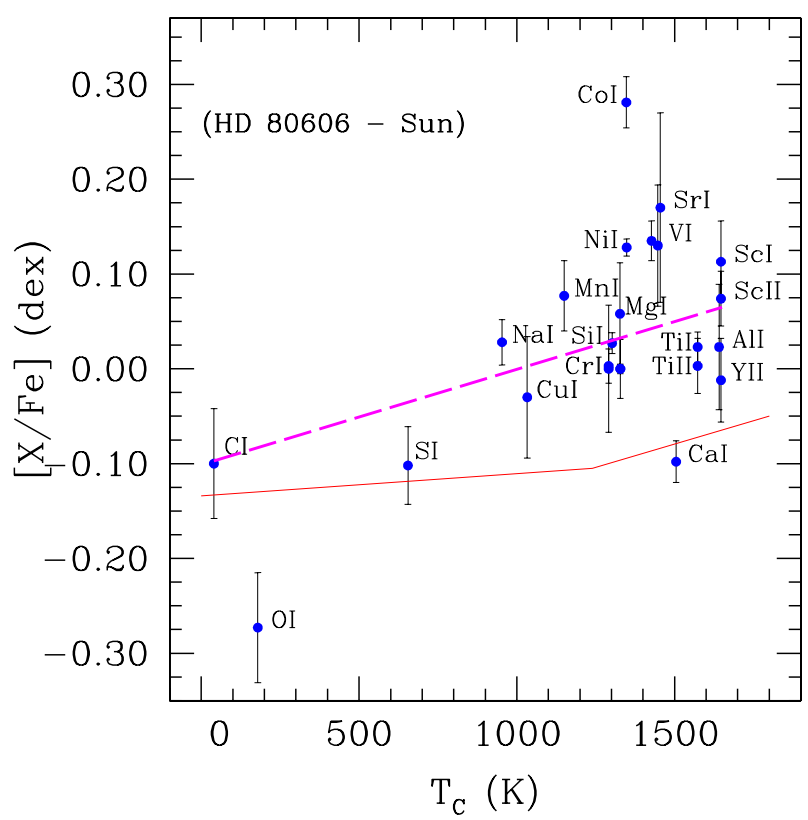

Fig. 5. Differential abundances (HD 80606 - Sun) vs. condensation temperature $T_{\mathrm{c}}$. The dashed line is a weighted linear fit to the differential abundance values, while the continuous line shows the solar-twins trend of Meléndez et al. (2009).

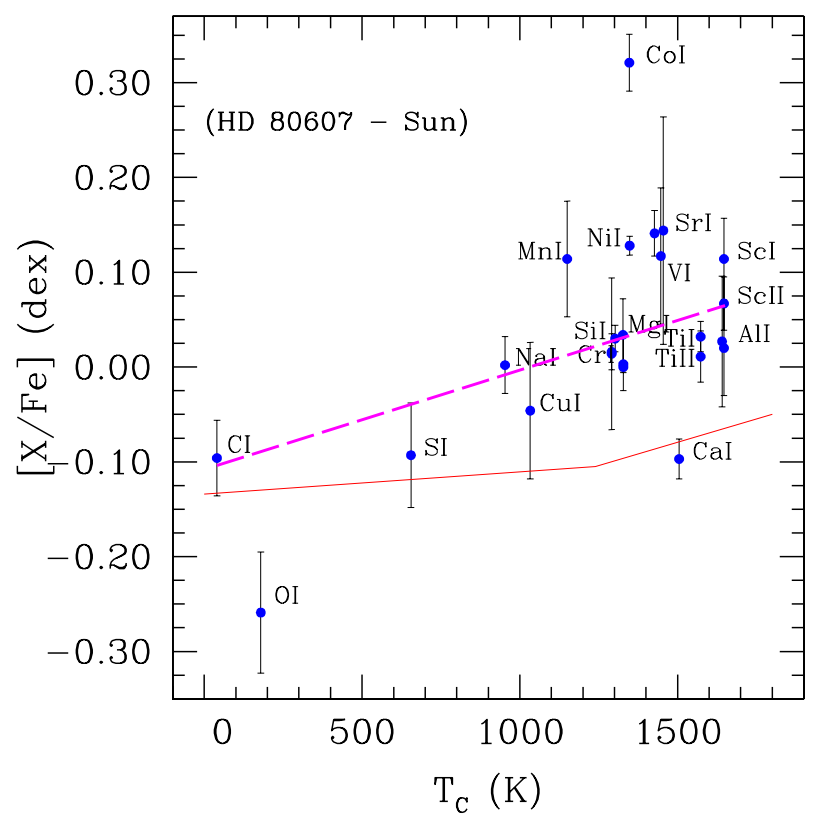

Fig. 6. Differential abundances (HD 80607 - Sun) vs. condensation temperature $T_{\mathrm{c}}$. The dashed line is a weighted linear fit to the differential abundance values, while the continuous line shows the solar-twins trend of Meléndez et al. (2009).

figures the solar-twins trend of M09 using a continuous line, vertically shifted to compare the slopes. We included a weighted linear fit ${ }^{10}$ to all abundance values, shown with dashed lines in Figs. 5 and 6. The slopes of the linear fits are similar to the trend of the solar-twins of M09 for the refractory elements.

In Figs. 5 and 6, the abundance of O I presents a low value compared to other volatile elements, while the abundances of Co I and Ca I seem to deviate from the general trend of the refractory elements (see also the next Figs. 7 and 8). For both

${ }^{10}$ We used as weight the inverse of the total abundance error $\sigma_{\text {Tот }}$. 
A\&A 582, A17 (2015)

Table 2. Differential abundances for the stars HD 80606 and HD 80607 relative to the Sun, and HD 80607 relative to HD 80606.

\begin{tabular}{|c|c|c|c|c|c|c|c|c|c|c|c|c|}
\hline \multirow[b]{2}{*}{ Element } & \multicolumn{4}{|c|}{ (HD 80606 - Sun) } & \multicolumn{4}{|c|}{ (HD 80607 - Sun) } & \multicolumn{4}{|c|}{ (HD 80607-HD 80606) } \\
\hline & {$[\mathrm{X} / \mathrm{Fe}]$} & $\sigma_{\mathrm{obs}}$ & $\sigma_{\text {par }}$ & $\sigma_{\mathrm{TOT}}$ & {$[\mathrm{X} / \mathrm{Fe}]$} & $\sigma_{\mathrm{obs}}$ & $\sigma_{\mathrm{par}}$ & $\sigma_{\mathrm{TOT}}$ & {$[\mathrm{X} / \mathrm{Fe}]$} & $\sigma_{\mathrm{obs}}$ & $\sigma_{\mathrm{par}}$ & $\sigma_{\mathrm{TOT}}$ \\
\hline$[\mathrm{C} \mathrm{I} / \mathrm{Fe}]$ & -0.040 & 0.000 & 0.057 & 0.058 & -0.036 & 0.000 & 0.039 & 0.040 & +0.004 & 0.000 & 0.028 & 0.028 \\
\hline & 193 & 0.041 & 0.041 & 58 & 79 & 0.057 & 0.029 & 64 & 14 & 0.031 & .020 & 037 \\
\hline$[\mathrm{Na} \mathrm{I} / \mathrm{Fe}]$ & 0.022 & 0.017 & 0.016 & 0.024 & -0.048 & 0.028 & 0.011 & 30 & 26 & 0.015 & .006 & 17 \\
\hline$[\mathrm{Mg} \mathrm{I} / \mathrm{Fe}]$ & 0.078 & 0.050 & 0.019 & 0.054 & 0.054 & 0.033 & 0.017 & 0.038 & 024 & 0.021 & 0.011 & 24 \\
\hline$[\mathrm{Al} \mathrm{I} / \mathrm{Fe}]$ & 0.003 & 0.064 & 0.016 & 0.066 & 0.007 & 0.068 & 0.012 & 0.069 & +0.004 & 0.007 & 0.009 & 0.012 \\
\hline $\mathrm{Fe}]$ & 0.027 & 0.010 & 0.002 & 0.011 & 0.030 & 0.012 & 0.003 & 0.014 & +0.003 & 0.004 & 0.002 & 0.005 \\
\hline & -0.052 & 0.032 & 0.026 & 0.041 & -0.043 & 0.050 & 0.021 & $0 .($ & +0.009 & 0.025 & 0.013 & 0.029 \\
\hline$[\mathrm{Ca} \mathrm{I} / \mathrm{Fe}]$ & -0.048 & 0.016 & 0.015 & 0.022 & -0.047 & 0.016 & 0.013 & 0.021 & +0.001 & 0.003 & 0.008 & 0.009 \\
\hline$[\mathrm{Sc} \mathrm{I} / \mathrm{Fe}]$ & 0.073 & 0.035 & 0.023 & 0.043 & 0.074 & 0.041 & 0.013 & $0 .($ & +0.002 & 0.006 & 0.009 & 0.011 \\
\hline$[\mathrm{Sc}$ & 0.034 & 0.014 & 0.025 & 0.029 & 0.027 & 0.017 & 0.021 & $0 .($ & -0.007 & 0.004 & 0.015 & 0.015 \\
\hline$[\mathrm{Ti}$ & 0.033 & 0.012 & 0.009 & 0.016 & 0.042 & 0.011 & 0.009 & $0 .($ & +0.008 & 0.005 & 0.004 & 0.007 \\
\hline [Ti II/Fe] & 0.013 & 0.022 & 0.019 & 0.029 & 0.021 & 0.020 & 0.017 & 0.027 & +0.008 & 0.014 & 0.012 & 0.019 \\
\hline & 0.085 & 0.016 & 0.013 & 0.021 & 0.091 & 0.019 & 0.013 & 0.024 & +0.006 & 0.006 & 0.008 & 0.011 \\
\hline$[\mathrm{Cr} \mathrm{I} / \mathrm{Fe}]$ & 0.003 & 0.014 & 0.011 & & 0.016 & & 0.010 & & +0.013 & 0.005 & 0.005 & 0.008 \\
\hline & 0.000 & 0.054 & 0.040 & 00 & 14 & & 0.038 & 30 & 014 & 0.016 & 0.023 & 0.029 \\
\hline $\mathrm{I} / \mathrm{Fe}]$ & -0.023 & 0.0 & 0.023 & 0. & 4 & $0 .($ & 0. & & 37 & 0. & 0. & 19 \\
\hline $\mathrm{Fe}]$ & 0.191 & 0.020 & 0.016 & 0.0 & 0.231 & 0.024 & 0.016 & 0.0 & +0.040 & 0.008 & 0.010 & 0.013 \\
\hline [Ni I/Fe] & 0.078 & 0.007 & 0.004 & 0.009 & 0.078 & 0.007 & 0.005 & 0.010 & -0.001 & 0.004 & 0.003 & 0.005 \\
\hline $\mathrm{I} / \mathrm{Fe}]$ & -0.070 & 0.050 & 0.040 & 0.064 & -0.086 & 0.060 & 0.040 & 0.072 & -0.016 & 0.020 & 0.025 & 0.032 \\
\hline & 0.120 & 0.050 & 0.086 & 0.100 & 0.094 & 0.060 & 0.106 & 0.120 & -0.026 & 0.020 & 0.055 & 0.058 \\
\hline & -0.002 & 0.028 & 0.034 & 0.0 & 0.030 & 0.027 & 0.042 & 0.050 & +0.032 & 0.009 & 0.025 & 0.026 \\
\hline$[\mathrm{Ba} \mathrm{II} / \mathrm{Fe}]$ & 0.190 & 0.050 & 0.040 & 0.064 & 0.177 & 0.060 & 0.040 & 0.072 & -0.013 & 0.020 & 0.025 & 0.032 \\
\hline
\end{tabular}

Notes. We also present the observational errors $\sigma_{\mathrm{obs}}$, errors due to stellar parameters $\sigma_{\text {par }}$, as well as the total error $\sigma_{\text {TOT. }}$.

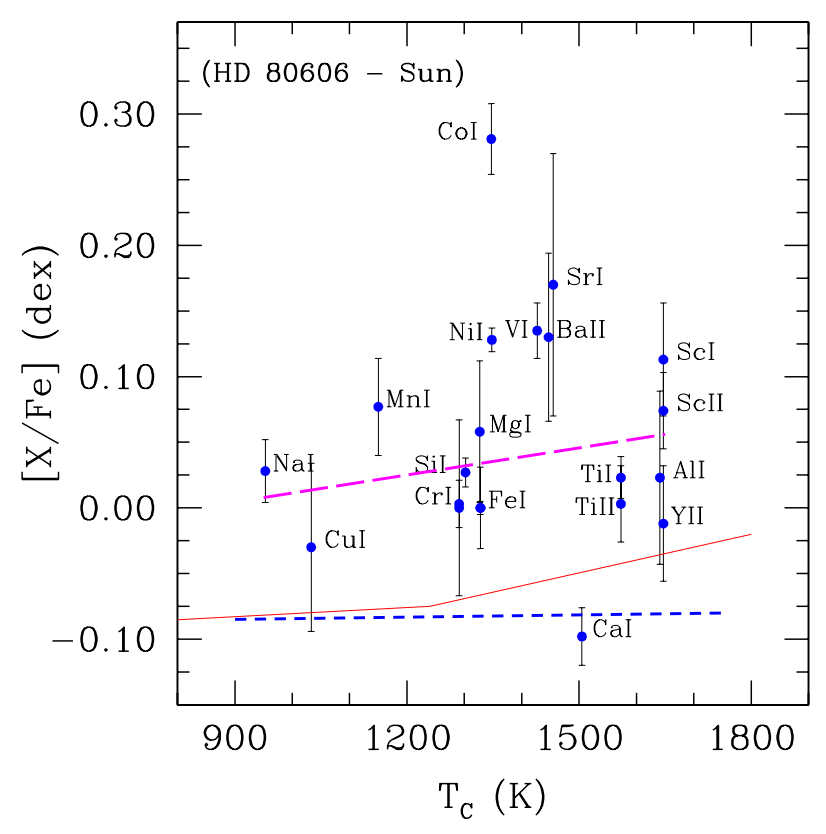

Fig. 7. Differential abundances (HD 80606 - Sun) vs. condensation temperature $T_{\mathrm{c}}$ for the refractory elements. The long-dashed line shows a weighted linear fit to the abundance values. The continuous and shortdashed lines correspond to the solar-twins trend of M09, and the solaranalogues with $[\mathrm{Fe} / \mathrm{H}] \sim+0.2$ dex from N09.

stars, we derived the $\mathrm{O} I$ abundance by measuring EWs of the O I triplet at $7771 \AA$ and applied NLTE corrections following Ramírez et al. (2007). As we noted previously, the NLTE corrections decrease the abundance in $\sim 0.11$ dex, However, even the LTE values seem to be relatively low; we do not find a clear reason for this. The forbidden [O I] lines at $6300.31 \AA$ and $6363.77 \AA$ are weak and slightly asymetric in our stars. Both [O I] lines are blended in the solar spectra: with two N I lines

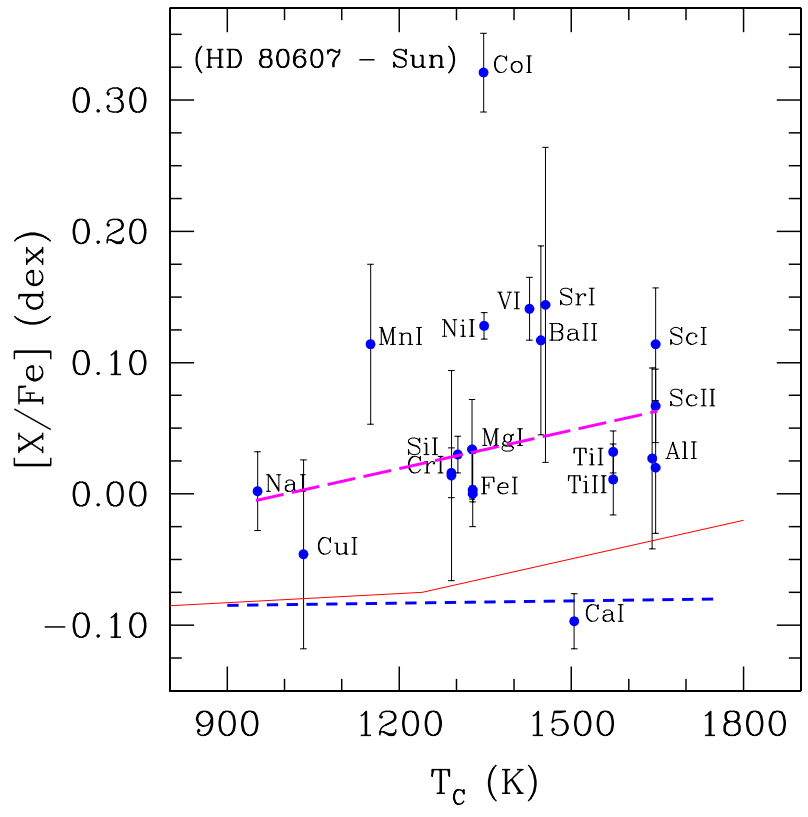

Fig. 8. Differential abundances (HD 80607 - Sun) vs. condensation temperature $T_{\mathrm{c}}$ for the refractory elements. The symbols are the same of Fig. 7.

in the red wing of [O I] $6300.31 \AA$ and with $\mathrm{CN}$ near [O I] $6363.77 \AA$ (Lambert 1978; Johansson et al. 2003; Bensby et al. 2004). Then, we prefer to avoid these weak [O I] lines in our calculation and only use the O I triplet. For the case of Co I, we take the HFS into account in the abundance calculation, however, NLTE effects could also play a role in the Co I lines of solartype stars (see e.g. Bergemann 2008; Bergemann et al. 2010). Mashonkina et al. (2007) studied NLTE effects in the Ca I lines of late-type stars and derived higher NLTE abundances than in LTE for most Ca I lines, using a model with $T_{\mathrm{eff}}=5500 \mathrm{~K}$ and 
$[\mathrm{Fe} / \mathrm{H}]=0$. For these stellar parameters, the corrections amount up to 0.08 dex with an average of $\sim 0.05 \mathrm{dex}$, however, we caution that these studies for Co I and $\mathrm{Ca}$ I do not include corrections for stars with $[\mathrm{Fe} / \mathrm{H}]>0$. Therefore, we excluded these species (O I, Co I and Ca I) from the calculation of the linear fits.

Ramírez et al. (2010, hereafter R10) studied the abundance results from six different abundance surveys and verified the findings of M09 regarding the $T_{\mathrm{c}}$ trends in the Sun and the terrestrial planet formation signature. They studied the possible dependence of the $T_{\mathrm{c}}$ trends with $[\mathrm{Fe} / \mathrm{H}]$, in particular, using the sample of Neves et al. (2009, hereafter N09). The authors show that the "solar anomaly" (i.e. the $T_{\mathrm{c}}$ trend for the refractory elements in the Sun) is also observed comparing the Sun with solar-analogues at both $[\mathrm{Fe} / \mathrm{H}] \sim-0.2 \mathrm{dex}$ and $[\mathrm{Fe} / \mathrm{H}] \sim 0.0$ dex. However, for an average metallicity of $[\mathrm{Fe} / \mathrm{H}] \sim+0.2$ dex, the solar analogues from N09 shows a $T_{\mathrm{c}}$ trend for refractories similar to the Sun (see e.g. their Fig. 7). R10 interpret this result suggesting that at high-metallicity values the probability of stars with and without $T_{\mathrm{c}}$ trends should be similar, and then they find that, on average, no general trend with $T_{\mathrm{c}}$ result for the refractory elements. The authors also propose that it may be possible to distinguish metal-rich stars that show and do not show the planet formation signature from the $T_{\mathrm{c}}$ slopes of the refractory elements. Then, given that HD 80606 and HD 80607 present high-metallicity values, it also seems reasonable to compare the refractories with the solar-analogue stars with $[\mathrm{Fe} / \mathrm{H}] \sim+0.2 \mathrm{dex}$ from N09.

The differential abundances of the refractory species are shown in Figs. 7 and 8. We include in these figures the trend of the solar-analogue stars with $[\mathrm{Fe} / \mathrm{H}] \sim+0.2$ dex from N09 using a short-dashed line, which shows a near horizontal tendency. The solar-twins $T_{\mathrm{c}}$ trend of M09 is also shown with a continuous line. The tendences of N09 and M09 are vertically shifted for comparison. A weighted linear fit to the refractory species of HD 80606 and HD 80607 is presented with a long-dashed line. The refractory elements does not seem to follow a horizontal trend like the sample of N09. The general trend of refractory species for both HD 80606 and HD 80607, are more similar to the solar-twins of M09 than to the solar-analogues stars with $[\mathrm{Fe} / \mathrm{H}] \sim+0.2 \mathrm{dex}$ from N09. The Sun is depleted in refractory elements compared to the solar-twins of M09, however, the solar-analogues with $[\mathrm{Fe} / \mathrm{H}] \sim+0.2$ dex from N09 present a similar $T_{\mathrm{c}}$ trend compared to the Sun, as shown by R10. Then, following a reasoning similar to M09 and R10, the stars HD 80606 and HD 80607 do not seem to be depleted in refractory elements with respect to solar twins, which is different for the case of the Sun. In other words, the terrestrial planet formation would have been less efficient in the stars of this binary system than in the Sun.

The line-by-line differential abundances between HD 80606 and HD 80607 greatly diminishes the errors in the calculation and GCE effects in the results because they have remarkably similar stellar parameters and the same (initial) chemical composition. In Fig. 9 we show the differential abundances of HD 80607 vs. $T_{\mathrm{c}}$ but using HD 80606 as the reference star. The continuous line in this figure presents the solar-twins trend of M09 (vertically shifted), while the long-dashed line is a weighted linear fit to the refractory elements. We included an horizontal line at 0.0 dex for reference.

Most elements present slightly higher abundance values in HD 80606 compared to HD 80607, with an average difference of $+0.010 \pm 0.019$ dex. In particular, the difference for the Fe I abundances is $+0.014 \pm 0.003$ dex, i.e. HD 80606 is slightly more metal-rich than HD 80607. From Fig. 9, the abundances

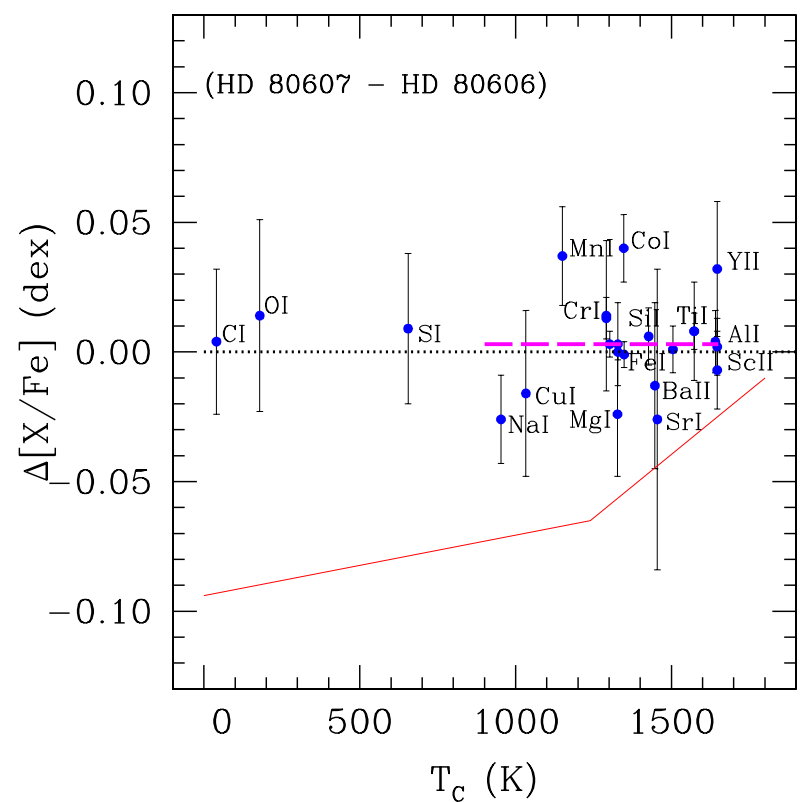

Fig. 9. Differential abundances (HD 80607-HD 80606) vs. condensation temperature $T_{\mathrm{c}}$. The long-dashed line is a weighted linear fit to the refractory species. The solar-twins trend of Meléndez et al. (2009) is shown with a continuous line. The horizontal line at $0.0 \mathrm{dex}$ is included for reference.

of the volatile does not seem to be different from the refractory elements. Their average abundances are $-0.005 \pm 0.005 \mathrm{dex}$ and $-0.011 \pm 0.005$ dex, i.e. almost the same within the errors. In Fig. 9, the slope of the differential abundances is $-1.20 \pm$ $16.5 \times 10^{-6} \mathrm{dex} / \mathrm{K}$ for the refractory elements. For comparison, the slope of refractories between the components of the binary system 16 Cyg results in $1.88 \pm 0.79 \times 10^{-5} \mathrm{dex} / \mathrm{K}$, and clearly shows a higher abundance in refractory than volatile elements (Tucci Maia et al. 2014). Then, although HD 80606 seems to present a slightly higher Fe I abundance than HD 80607, there is no clear difference between refractory and volatile elements nor is there a significative trend with $T_{\mathrm{c}}$. This would imply that there is no clear evidence of terrestrial planet formation in this binary system. Similarly, Liu et al. (2014) did not find a trend with $T_{\mathrm{c}}$ in the binary system HAT-P-1 and concluded that the presence of a giant planet does not neccesarily introduce a chemical signature in their host stars. This is in line with some previous literature works, who propose that the presence of close-in giant planets might prevent the formation of terrestrial planets (Meléndez et al. 2009; Steffen et al. 2012). For the case of eccentric giant planets, numerical simulations also found that the early dynamical evolution of giant planets clear out most of the terrestrial planets in the inner zone (Veras \& Armitage 2005, 2006; Raymond et al. 2011).

\subsection{A planet around HD 80607 ?}

Up to now, there is no planet detected around HD 80607. The photometry of HD 80607 is relatively flat, i.e. a transit-like event is not observed (Fossey et al. 2009; Pont et al. 2009). To our knowledge, this object is not included in the current radial velocity surveys.

However, given the abundance results of this study and the confirmed presence of a giant planet (with very high eccentricity) only around HD 80606, we can speculate about a possible planet formation scenario in this binary system. The occurrence of planets was fit by Fischer \& Valenti (2005) 
using a power law as a function of the metallicity: $P=0.03$ $\left(N_{\mathrm{Fe}} / N_{\mathrm{H}}\right)^{2} /\left(N_{\mathrm{Fe}} / N_{\mathrm{H}}\right)_{\odot}^{2}$. Then, the probability increases by a factor of 5 when the $\mathrm{Fe}$ abundance increases from $[\mathrm{Fe} / \mathrm{H}]=0.0$ dex to $[\mathrm{Fe} / \mathrm{H}]=0.3$ dex. This high probability, together with the fact that HD 80606 already hosts a giant planet and given the very similar stellar parameters with HD 80607, suggests that the giant planet formation process in HD 80607 could be also a very plausible hypothesis. The metals missing in HD 80607 compared to HD 80606 could possibly have been used to form this (hypothetic) giant planet. Tucci Maia et al. (2014) make a similar suggestion to explain the slightly different metallicities between the components of the binary system 16 Cyg. Moreover, there are binary systems in which each component hosts a planet and the metallicity results were slightly different between their stars, such as in the system XO-2 (Damasso et al. 2015). Then, probably because of the mutual interactions in this binary system, HD $80606 \mathrm{~b}$ became one of the most eccentric planets to date (see e.g. Wu \& Murray 2003), while the HD 80607 system may have had its giant planet ejected. In fact, the possible companion around HD 80607 could be an ejected or maybe an undetected (such as a long period) planet. We stress, however, that this is only a speculative comment and should be taken with caution.

Previous works showed that the global frequency of planets in wide binaries is not statistically different from that of planets in single stars, with no significant dependence of the binary separation (Bonavita \& Desidera 2007). Also, the properties of planets in wide binaries are compatible with those of planets orbiting single stars, except for a possible increase of high-eccentricity planets (Desidera \& Barbieri 2007). However, the presence of closer stellar companions with separation 100-300 AU could modify the evolution of giant planets around binary components (Desidera \& Barbieri 2007).

More recently, Wang et al. (2015) studied 84 KOIs (Kepler Object of Interest) with at least one gas giant planet detected within $1 \mathrm{AU}$ and a control sample of field stars in the solar neighborhood. The authors found a dependence of the stellar multiplicity rate (MR) as a function of the stellar separation a. They derived MRs of $\sim 0 \%, \sim 34 \%$, and $>34 \%$ for binary separations of $a<20 \mathrm{AU}, 20 \mathrm{AU}<a<200 \mathrm{AU}$, and $a>200 \mathrm{AU}$, respectively. In other words, no stellar companion has been found within $20 \mathrm{AU}$ for Kepler stars with gas giant planets, while gas giant planet formation is not significantly affected by stellar companions beyond $200 \mathrm{AU}$. Then, this work shows that the binary separation plays a role in close binaries rather than in wide binaries, such as HD 80606 ( $a \sim 1000$ AU). This is in agreement with Zuckerman (2014), who found that the presence of a wide stellar companion ( $a \geq 1000 \mathrm{AU}$ ) does not diminish the likelihood of a wide-orbit planetary system.

Wang et al. (2015) also studied the possible physical differences between the components of binaries hosting planets. They suggest that the stellar companions of host stars with a planet period $P>70 \mathrm{~d}$ tend to be fainter than the shorter-period counterparts. However, they caution that this apparent effect may be due to a lack of sensivity for fainter stellar companions and suggest more follow-up observations to support or disprove it.

Using numerical simulations, Wu \& Murray (2003) suggest that the high eccentricity of the planet HD $80606 \mathrm{~b}$ is probably due to the influence of the companion HD 80607 through a Kozai mechanism ${ }^{11}$ combined with a tidal dissipation. On the other hand, Kaib et al. (2013) showed a possible variable nature

\footnotetext{
11 The Kozai mechanism are oscilations in the eccentricity and inclination of a planet due to the presence of a remote stellar companion; see e.g. Kozai (1962).
}

of wide binaries due to the Milky Way tidal field, including a reshape of their planetary systems. In this scenario, they obtained an instability fraction (i.e. number of planetary ejections within 10 Gyr of evolution) depending on the binary's mass and separation. Using the binary parameters of HD 80606, they obtained a fraction of $\sim 50 \%$ (see their Fig. 2). Although these simulations do not include the possibility of a planet around HD 80607, they showed that the planetary configuration in this binary system could be strongly affected, and the possible ejection of a planet could not be totally ruled out.

\section{Conclusions}

Following the aims of this study, we performed a high-precision differential abundance determination in both components of the remarkable binary system HD 80606-HD 80607, in order to detect a possible signature of terrestrial planet formation. Both stars present very similar stellar parameters, which greatly diminishes the errors in the abundance determination and GCE effects. The star HD 80606 hosts a giant (high-eccentricity) planet while there is no planet detected around HD 80607 . First, we derived stellar parameters and differential abundances of both stars using the Sun as the reference star. We compared the possible temperature condensation $T_{\mathrm{c}}$ trends of the stars with the solartwins trend of Meléndez et al. (2009) and then compared the temperature condensation trend with a sample of solar-analogue stars with $[\mathrm{Fe} / \mathrm{H}] \sim+0.2$ dex from Neves et al. (2009). Our calculation included NLTE corrections for O I and Ba II as well as GCE corrections for all chemical species. From these comparisons, we concluded that the stars HD 80606 and HD 80607 do not seem to be depleted in refractory elements, which differs from the case of the Sun (Meléndez et al. 2009). In other words, the terrestrial planet formation would have been less efficient in the stars of this binary system than in the Sun.

Then, we also compared HD 80607 differentially, using HD 80606 as the reference star. HD 80606 was slightly more metal-rich than HD 80607 by $+0.014 \pm 0.003$ dex. However, we do not find a clear difference between refractory and volatile elements nor a significative trend with $T_{\mathrm{c}}$ between both stars. In comparing the stars to each other, the lack of a trend for refractory elements with $T_{\mathrm{c}}$ implies that the presence of a giant planet does not necessarily imprint a chemical signature on its host star, which is similar to the result of Liu et al. (2014) for the binary system HAT-P-1. This is in agreement with Meléndez et al. (2009), who suggest that the presence of close-in giant planets might prevent the formation of terrestrial planets. Finally, we speculate about a possible (ejected or non-detected) planet around HD 80607 . We strongly encourage high-precision abundance studies in binary systems with similar components, which is a crucial tool for helping to detect the possible chemical pattern of the planet formation process.

Acknowledgements. We thank the anonymous referee for constructive comments that greatly improved the paper. The authors wish to recognize and acknowledge the very significant cultural role and reverence that the summit of Mauna Kea has always had within the indigenous Hawaiian community. We are most fortunate to have the opportunity to conduct observations from this mountain. The authors also thank Drs. R. Kurucz and C. Sneden for making their codes available to us.

\section{References}

Bakos, G. A., Noyes, R. W., Kovács, G., et al. 2007, ApJ, 656, 552 Bedell, M., Meléndez, J., Bean, J. L., et al. 2014, ApJ, 795, 23 Beirao, P., Santos, N. C., Israelian, G., \& Mayor, M. 2005, A\&A, 438, 251 
Bensby, T., Feltzing, S., \& Lundström, I. 2004, A\&A, 415, 155

Bergemann, M. 2008, Phys. Scr. T., 133, 014013

Bergemann, M., Pickering, J., \& Gehren, T. 2010, MNRAS, 401, 1334

Bonavita, M., \& Desidera, S. 2007, A\&A, 468, 721

Castelli, F., \& Kurucz, R. 2003, New Grids of ATLAS9 Model Atmospheres, held at Uppsala, Sweden, 2002, eds. N. Piskunov, W. W. Weiss, \& D. F. Gray, IAU Symp., 210, A20

Cochran, William, D., Hatzes, A. P., Butler, R. P., \& Marcy, G. W. 1997, ApJ, 483, 457

Damasso, M., Biazzo, K., Bonomo, A., et al. 2015, A\&A, 575, A111

Desidera, S., \& Barbieri, M. 2007, A\&A, 462, 345

Desidera, S., Gratton, R. G., Scuderi, S., et al. 2004, A\&A, 420, 683

Desidera, S., Gratton, R. G., Lucatello, S., \& Claudi, R. U. 2006, A\&A, 454, 581

Dommanget, J., \& Nys, O. 2002, The Catalog of the Components of Double and Multiple Stars (CCDM), Second Edition, Vizier Online Data Catalog I/274

Ecuvillon, A., Israelian, G., Santos, N. C., et al. 2006, A\&A, 445, 633

Epstein, C., Johnson, J., Dong, S., et al. 2010, ApJ, 709, 447

Fabbian, D., Asplund, M., Barklem, P., Carlsson, M., \& Kiselman, D. 2009, A\&A, 500, 1221

Fischer, D., \& Valenti, J. 2005, AJ, 622, 1102

Fossey, S., Waldmann, I., \& Kipping, D. 2009, MNRAS, 396, L16

Gilli, G., Israelian, G., Ecuvillon, A., Santos, N. C., \& Mayor, M. 2006, A\&A, 449,723

Gonzalez, G., \& Laws, C. 2007, MNRAS, 378, 1141

González Hernández, J., Delgado Mena, E., Sousa, S. G., et al. 2013, A\&A, 552, A6

Gratton, R. G., Bonanno, G., Claudi, R. U., et al. 2001, A\&A, 377, 123

Jofré, E., Petrucci, R., Saffe, C., et al. 2015, A\&A, 574, A50

Johansson, S., Litzén, U., Lundberg, H., \& Zhang, Z. 2003, ApJ, 584, L107

Kaib, N., Raymond, S., \& Duncan, M. 2013, Nature, 493, 381

King, R., Parker, R., Patience, J., \& Goodwin, S. 2012, MNRAS, 421, 2025

Koleva, M., \& Vazdekis, A. 2012, A\&A, 538, A143

Korotin, S., Mishenina, T., Gorbaneva, T., \& Soubiran, C. 2011, MNRAS, 415, 2093

Kozai, Y. 1962, AJ, 67, 591

Kratter, K. M. 2011, in Evolution of Compact Binaries, eds. L. Schmidtobreick, M. R. Schreiber, \& C. Tappert (San Francisco, CA: ASP), ASP Conf. Ser., 447, 47

Kurucz, R. L. 1993, ATLAS9 Stellar Atmosphere Programs and $2 \mathrm{~km} \mathrm{~s}^{-1}$ grid, Kurucz CD-ROM No. 13 (Cambridge, MA: Smithsonian Astrophysical Observatory)

Kurucz, R., \& Bell, B. 1995, Atomic Line Data, Kurucz CD-ROM No. 23 (Cambridge, MA: Smithsonian Astrophysical Observatory)
Lambert, D. L. 1978, MNRAS, 182, 249

Laughlin, G., Deming, D., Langton, J., et al. 2009, Nature, 457, L562

Laws, C., \& Gonzalez, G. 2001, ApJ, 553, L405

Liu, F., Asplund, M., Ramírez, I., Yong, D., \& Meléndez, J. 2014, MNRAS, 442. L51

Lodders, K. 2003, AJ, 591, 1220

Mashonkina, L., Korn, A., \& Przybilla. N. 2007, A\&A, 461, 261

Meléndez, J., Asplund, M., Gustafsson, B., \& Yong, D. 2009, AJ, 704, L66

Meléndez, J., Ramírez, I., Karakas, A., et al. 2014, AJ, 791, 14

Mollá, M., Cavichia, O., \& Gibson, B. 2015, MNRAS, 451, 3693

Mortier, A., Santos, N. C., Sousa, S. G., et al. G. 2013, A\&A, 558, A106

Moutou, C., Hébrard, G., Bouchy, F., et al. 2009, A\&A, 498, L5

Naef, D., Latham, D. W., Mayor, M., et al. 2001, A\&A, 375, L27

Neves, V., Santos, N. C., Sousa, S. G., Correia, A. C. M., \& Israelian, G. 2009, A\&A, 497, 563

Pont, F., Hébrand, G., Irwin, J., et al. 2009, A\&A, 502, 695

Ramírez, I., Allende Prieto, C., \& Lambert, D. 2007, A\&A, 465, 271

Ramírez, I., Asplund, M., Baumann, P., Meléndez, J., \& Bensby, T. 2010, A\&A, 521, A33

Ramírez, I., Meléndez, J., Cornejo, D., Roederer, I., \& Fish, J. 2011, AJ, 740, 76

Raymond, S., Armitage, P., Moro-Martín, A., et al. 2011, A\&A, 530, A62

Reiputh, B., \& Mikkola, S. 2012, Nature, 492, 221

Reiputh, B., Guimaraes, M., Connelley, M., \& Bally, J. 2007, AJ, 134, 2272

Saffe, C. 2011, Rev. Mex. Astron. Astrofis., 47, 3

Saffe, C., \& Levato, H. 2014, A\&A, 562, A128

Santos, N. C., Israelian, G., \& Mayor, M. 2004, A\&A, 415, 1153

Santos, N. C., Israelian, G., Mayor, M., et al. 2005, A\&A, 437, 1127

Schuler, S., Cunha, K., Smith, V., et al. 2011, ApJ, 737, L32

Sneden, C. 1973, ApJ, 184, 839

Sousa, S. G., Santos, N. C., Mayor, M., et al. 2008, A\&A, 487, 373

Steffen, J., Ragozzine, D., Fabycky, D., et al. 2012, Proc. Nat. Acad. Sci. USA, 109, 7982

Takeda, Y. 2003, A\&A, 402, 343

Takeda, Y. 2005, PASJ, 57, 83

Tayouchi, D., \& Chiba, M. 2014, AJ, 788, 89

Teske, J., Schuler, S., Cunha, K., Smith, V., \& Griffith, C. 2013, ApJ, 768, L12

Tucci Maia, M., Meléndez, J., \& Ramírez, I. 2014, ApJ, 790, L25

Udry, S., Mayor, M., Benz, W., et al. 2006, A\&A, 447, 361

Veras, D., \& Armitage, P. 2005, ApJ, 620, L111

Veras, D., \& Armitage, P. 2006, ApJ, 645, 1509

Vogt, N., Schmidt, T., Neuhäuser, R., et al. 2012, A\&A, 546, A63

Wang, J., Fischer, D., Horch, E., \& Xie, J. 2015, ApJ, 806, 248

Wu, Y., \& Murray, N. 2003, ApJ, 589, 605

Zuckerman, B. 2014, ApJ, 791, L27 
Table 1. Line list used in this work.

\begin{tabular}{|c|c|c|c|c|c|c|}
\hline Element & $\begin{array}{c}\lambda \\
{[\AA]}\end{array}$ & $\begin{array}{c}\mathrm{EP} \\
{[\mathrm{eV}]}\end{array}$ & $\begin{array}{r}\log g f \\
{[\mathrm{dex}]}\end{array}$ & $\begin{array}{c}E W_{1} \\
{[\mathrm{m \AA}]}\end{array}$ & $\begin{array}{c}E W_{2} \\
{[\mathrm{~m} \AA]}\end{array}$ & $\begin{array}{c}E W_{\text {Sun }} \\
{[\mathrm{m} \AA]}\end{array}$ \\
\hline 6.00 & 5052.167 & 7.680 & -1.240 & 42.0 & 39.4 & 33.6 \\
\hline 6.00 & 6587.610 & 8.540 & -1.050 & 16.6 & 14.8 & 12.1 \\
\hline 8.00 & 7771.944 & 9.150 & 0.370 & 65.7 & 58.4 & 66.9 \\
\hline 8.00 & 7774.166 & 9.150 & 0.220 & 61.5 & 59.3 & 62.1 \\
\hline 8.00 & 7775.388 & 9.150 & 0.000 & 49.8 & 47.2 & 45.0 \\
\hline 11.00 & 4751.822 & 2.100 & -2.080 & 36.7 & 38.5 & 15.7 \\
\hline 11.00 & 5148.838 & 2.100 & -2.040 & 31.0 & 31.7 & 13.8 \\
\hline 11.00 & 6154.225 & 2.100 & -1.550 & 75.3 & 80.6 & 39.2 \\
\hline 11.00 & 6160.747 & 2.100 & -1.250 & 91.8 & 94.1 & 56.9 \\
\hline 12.00 & 4730.040 & 4.340 & -2.390 & 112.1 & 108.7 & 68.6 \\
\hline 12.00 & 5711.088 & 4.340 & -1.730 & 147.0 & 144.6 & 106.6 \\
\hline 12.00 & 6318.717 & 5.110 & -1.950 & 62.1 & 63.8 & 37.3 \\
\hline 12.00 & 6319.236 & 5.110 & -2.160 & 52.1 & 51.5 & 24.2 \\
\hline 13.00 & 5557.070 & 3.140 & -2.210 & 25.5 & 25.6 & 13.4 \\
\hline 13.00 & 6696.018 & 3.140 & -1.480 & 62.3 & 64.7 & 36.0 \\
\hline 13.00 & 6698.667 & 3.140 & -1.780 & 47.0 & 48.6 & 20.8 \\
\hline 14.00 & 5488.983 & 5.610 & -1.690 & 38.1 & 36.8 & 18.5 \\
\hline 14.00 & 5517.540 & 5.080 & -2.500 & 24.6 & 23.6 & 12.2 \\
\hline 14.00 & 5645.611 & 4.930 & -2.040 & 56.7 & 56.7 & 35.8 \\
\hline 14.00 & 5665.554 & 4.920 & -1.940 & 65.0 & 65.5 & 39.3 \\
\hline 14.00 & 5684.484 & 4.950 & -1.550 & 81.2 & 80.6 & 61.0 \\
\hline 14.00 & 5690.425 & 4.930 & -1.770 & 67.7 & 67.6 & 48.5 \\
\hline 14.00 & 5701.104 & 4.930 & -1.950 & 58.6 & 56.1 & 40.3 \\
\hline 14.00 & 5753.640 & 5.620 & -1.330 & 71.6 & 72.7 & 43.5 \\
\hline 14.00 & 5772.145 & 5.082 & -1.653 & 74.1 & 74.7 & 51.8 \\
\hline 14.00 & 5793.073 & 4.930 & -1.960 & 64.7 & 62.2 & 42.9 \\
\hline 14.00 & 5948.540 & 5.080 & -1.208 & 108.8 & 108.3 & 84.4 \\
\hline 14.00 & 6125.021 & 5.610 & -1.500 & 51.2 & 49.6 & 31.7 \\
\hline 14.00 & 6145.015 & 5.620 & -1.410 & 59.4 & 58.8 & 38.7 \\
\hline 14.00 & 6195.460 & 5.870 & -1.666 & 33.8 & 34.2 & 15.2 \\
\hline 14.00 & 6243.823 & 5.620 & -1.270 & 61.8 & 59.2 & 43.9 \\
\hline 14.00 & 6244.476 & 5.620 & -1.320 & 71.4 & 70.4 & 45.4 \\
\hline 14.00 & 6741.630 & 5.980 & -1.650 & 28.6 & 27.8 & 15.2 \\
\hline 14.00 & 7034.903 & 5.870 & -0.780 & 81.6 & 82.4 & 62.8 \\
\hline 14.00 & 7405.770 & 5.614 & -0.720 & 112.3 & 111.8 & 88.7 \\
\hline 16.00 & 4695.443 & 6.530 & -1.830 & 12.0 & 12.6 & 8.2 \\
\hline 16.00 & 6046.000 & 7.870 & -0.150 & 28.4 & 24.8 & 20.3 \\
\hline 16.00 & 6052.656 & 7.870 & -0.400 & 17.8 & 16.9 & 13.2 \\
\hline 16.00 & 6743.540 & 7.870 & -0.600 & 12.6 & 10.8 & 9.7 \\
\hline 20.00 & 5260.387 & 2.520 & -1.720 & 52.2 & 54.1 & 32.5 \\
\hline 20.00 & 5261.710 & 2.520 & -0.680 & 127.8 & 131.5 & 100.6 \\
\hline 20.00 & 5512.980 & 2.930 & -0.460 & 114.2 & 116.0 & 83.8 \\
\hline 20.00 & 5590.114 & 2.520 & -0.570 & 110.9 & 113.6 & 92.8 \\
\hline 20.00 & 5867.562 & 2.930 & -1.570 & 42.5 & 43.5 & 23.5 \\
\hline 20.00 & 6156.020 & 2.520 & -2.497 & 19.8 & 19.8 & 8.7 \\
\hline 20.00 & 6161.297 & 2.520 & -1.270 & 82.9 & 84.9 & 59.5 \\
\hline 20.00 & 6166.439 & 2.520 & -1.140 & 94.1 & 95.8 & 69.6 \\
\hline 20.00 & 6169.550 & 2.520 & -0.580 & 139.9 & 141.8 & 108.7 \\
\hline 20.00 & 6455.598 & 2.520 & -1.340 & 80.3 & 82.6 & 55.2 \\
\hline 20.00 & 6471.662 & 2.530 & -0.690 & 112.6 & 115.6 & 91.0 \\
\hline 20.00 & 6499.650 & 2.520 & -0.820 & 102.9 & 105.2 & 85.5 \\
\hline 21.00 & 4743.821 & 1.450 & 0.350 & 24.9 & 26.8 & 9.2 \\
\hline 21.00 & 5081.570 & 1.450 & 0.300 & 25.0 & 27.3 & 7.4 \\
\hline 21.00 & 5520.497 & 1.860 & 0.550 & 16.9 & 17.9 & 6.1 \\
\hline 21.00 & 5671.821 & 1.450 & 0.550 & 40.2 & 42.6 & 14.7 \\
\hline 21.10 & 5657.870 & 1.510 & -0.300 & 77.6 & 75.6 & 65.7 \\
\hline 21.10 & 5669.055 & 1.500 & -1.200 & 48.6 & 47.4 & 36.4 \\
\hline 21.10 & 5684.190 & 1.510 & -0.950 & 50.9 & 49.9 & 37.7 \\
\hline 21.10 & 6245.630 & 1.510 & -1.030 & 49.2 & 48.6 & 35.2 \\
\hline 21.10 & 6279.760 & 1.500 & -1.200 & 42.8 & 40.9 & 30.1 \\
\hline 21.10 & 6320.843 & 1.500 & -1.850 & 14.3 & 13.7 & 7.6 \\
\hline 21.10 & 6604.578 & 1.360 & -1.150 & 52.5 & 52.0 & 35.5 \\
\hline
\end{tabular}

Notes. The columns present the element, wavelength $\lambda$, excitation potential (EP), $\log g f$, equivalent widths of HD 80606, HD 80607, and Sun $\left(E W_{1}, E W_{2}\right.$, and $\left.E W_{\text {Sun }}\right)$. The abundances of lines without EWs are measured using synthetic spectra. 
Table 1. continued.

\begin{tabular}{|c|c|c|c|c|c|c|}
\hline Element & $\begin{array}{c}\lambda \\
{[\AA]} \\
{[\AA}\end{array}$ & $\begin{array}{c}\mathrm{EP} \\
{[\mathrm{eV}]}\end{array}$ & $\begin{array}{r}\log g f \\
{[\mathrm{dex}]}\end{array}$ & $\begin{array}{c}E W_{1} \\
{[\mathrm{~m} \AA]}\end{array}$ & $\begin{array}{l}E W_{2} \\
{[\mathrm{~m} \AA]}\end{array}$ & $\begin{array}{c}E W_{\text {Sun }} \\
{[\mathrm{m} \AA]}\end{array}$ \\
\hline 22.00 & 4617.280 & 1.750 & 0.450 & 85.6 & 88.1 & 64.1 \\
\hline 22.00 & 4645.190 & 1.730 & -0.670 & 40.8 & 44.8 & 21.7 \\
\hline 22.00 & 4656.470 & 0.000 & -1.310 & 91.4 & 95.6 & 68.4 \\
\hline 22.00 & 4758.120 & 2.250 & 0.430 & 61.4 & 63.2 & 43.0 \\
\hline 22.00 & 4759.272 & 2.260 & 0.510 & 66.3 & 67.6 & 47.0 \\
\hline 22.00 & 4778.258 & 2.240 & -0.220 & 35.3 & 35.3 & 15.4 \\
\hline 22.00 & 4820.410 & 1.500 & -0.440 & 66.7 & 69.2 & 43.0 \\
\hline 22.00 & 4999.500 & 0.830 & 0.270 & 135.8 & 138.4 & 104.5 \\
\hline 22.00 & 5022.871 & 0.830 & -0.430 & 93.7 & 96.4 & 70.9 \\
\hline 22.00 & 5024.850 & 0.820 & -0.560 & 98.5 & 100.7 & 70.0 \\
\hline 22.00 & 5039.960 & 0.020 & -1.200 & 100.7 & 101.0 & 76.2 \\
\hline 22.00 & 5071.490 & 1.460 & -0.800 & 57.0 & 57.2 & 27.7 \\
\hline 22.00 & 5147.479 & 0.000 & -2.010 & 59.9 & 64.1 & 34.1 \\
\hline 22.00 & 5219.700 & 0.020 & -2.240 & 59.7 & 64.5 & 29.1 \\
\hline 22.00 & 5471.200 & 1.440 & -1.400 & 21.9 & 25.2 & 7.9 \\
\hline 22.00 & 5490.150 & 1.460 & -0.930 & 42.7 & 46.7 & 21.0 \\
\hline 22.00 & 5689.459 & 2.300 & -0.360 & 29.0 & 31.1 & 11.5 \\
\hline 22.00 & 5739.464 & 2.250 & -0.600 & 20.2 & 22.1 & 6.3 \\
\hline 22.00 & 5766.330 & 3.290 & 0.326 & 22.3 & 23.1 & 9.0 \\
\hline 22.00 & 5866.452 & 1.070 & -0.840 & 76.7 & 79.6 & 47.6 \\
\hline 22.00 & 6064.630 & 1.050 & -1.959 & 25.6 & 27.1 & 7.8 \\
\hline 22.00 & 6091.174 & 2.270 & -0.420 & 35.5 & 37.9 & 14.7 \\
\hline 22.00 & 6126.217 & 1.070 & -1.420 & 46.2 & 49.5 & 22.4 \\
\hline 22.00 & 6258.104 & 1.440 & -0.350 & 79.2 & 82.3 & 50.4 \\
\hline 22.00 & 6303.753 & 1.443 & -1.509 & 24.5 & 25.7 & 8.0 \\
\hline 22.00 & 6312.234 & 1.460 & -1.496 & 20.5 & 23.6 & 6.8 \\
\hline 22.00 & 6599.104 & 0.900 & -2.029 & 27.2 & 29.6 & 8.8 \\
\hline 22.00 & 6743.130 & 0.899 & -1.630 & 43.2 & 47.1 & 17.8 \\
\hline 22.00 & 7949.150 & 1.500 & -1.456 & 29.4 & 32.2 & 8.2 \\
\hline 22.10 & 4636.330 & 1.160 & -3.150 & 30.5 & 27.3 & 17.6 \\
\hline 22.10 & 4779.985 & 2.048 & -1.260 & 72.2 & 76.3 & 65.2 \\
\hline 22.10 & 4798.532 & 1.080 & -2.670 & 53.2 & 52.4 & 42.6 \\
\hline 22.10 & 4865.611 & 1.120 & -2.810 & 55.9 & 54.2 & 40.7 \\
\hline 22.10 & 4911.193 & 3.120 & -0.540 & 64.1 & 64.1 & 53.3 \\
\hline 22.10 & 5005.160 & 1.570 & -2.720 & 31.0 & 31.5 & 19.6 \\
\hline 22.10 & 5418.767 & 1.580 & -2.110 & 60.5 & 59.1 & 49.4 \\
\hline 23.00 & 4875.442 & 0.040 & -3.375 & & & \\
\hline 23.00 & 4875.454 & 0.040 & -2.260 & & & \\
\hline 23.00 & 4875.461 & 0.040 & -2.964 & & & \\
\hline 23.00 & 4875.468 & 0.040 & -1.420 & & & \\
\hline 23.00 & 4875.471 & 0.040 & -2.064 & & & \\
\hline 23.00 & 4875.477 & 0.040 & -2.742 & & & \\
\hline 23.00 & 4875.483 & 0.040 & -1.561 & & & \\
\hline 23.00 & 4875.485 & 0.040 & -2.010 & & & \\
\hline 23.00 & 4875.491 & 0.040 & -2.617 & & & \\
\hline 23.00 & 4875.495 & 0.040 & -1.725 & & & \\
\hline 23.00 & 4875.497 & 0.040 & -2.032 & & & \\
\hline 23.00 & 4875.502 & 0.040 & -2.566 & & & \\
\hline 23.00 & 4875.505 & 0.040 & -1.923 & & & \\
\hline 23.00 & 4875.506 & 0.040 & -2.123 & & & \\
\hline 23.00 & 4875.509 & 0.040 & -2.596 & & & \\
\hline 23.00 & 4875.511 & 0.040 & -2.178 & & & \\
\hline 23.00 & 4875.511 & 0.040 & -2.311 & & & \\
\hline 23.00 & 4875.515 & 0.040 & -2.566 & & & \\
\hline 23.00 & 5703.555 & 1.050 & -0.777 & & & \\
\hline 23.00 & 5703.569 & 1.050 & -0.993 & & & \\
\hline 23.00 & 5703.569 & 1.050 & -1.403 & & & \\
\hline 23.00 & 5703.580 & 1.050 & -1.242 & & & \\
\hline 23.00 & 5703.580 & 1.050 & -1.276 & & & \\
\hline 23.00 & 5703.581 & 1.050 & -2.268 & & & \\
\hline 23.00 & 5703.589 & 1.050 & -1.250 & & & \\
\hline 23.00 & 5703.589 & 1.050 & -1.715 & & & \\
\hline 23.00 & 5703.590 & 1.050 & -1.840 & & & \\
\hline 23.00 & 5703.596 & 1.050 & -1.414 & & & \\
\hline 23.00 & 5703.596 & 1.050 & -1.590 & & & \\
\hline
\end{tabular}


Table 1. continued.

\begin{tabular}{|c|c|c|c|c|c|c|}
\hline Element & $\begin{array}{c}\lambda \\
{[\AA]}\end{array}$ & $\begin{array}{c}\mathrm{EP} \\
{[\mathrm{eV}]}\end{array}$ & $\begin{array}{r}\log g f \\
{[\operatorname{dex}]}\end{array}$ & $\begin{array}{c}E W_{1} \\
{[\mathrm{m \AA}]}\end{array}$ & $\begin{array}{c}E W_{2} \\
{[\mathrm{~m} \AA]}\end{array}$ & $\begin{array}{l}E W_{\text {Sun }} \\
{[\mathrm{m} \AA]}\end{array}$ \\
\hline 23.00 & 5703.601 & 1.050 & -1.414 & & & \\
\hline 23.00 & 5727.008 & 1.080 & -0.693 & & & \\
\hline 23.00 & 5727.016 & 1.080 & -1.701 & & & \\
\hline 23.00 & 5727.022 & 1.080 & -3.003 & & & \\
\hline 23.00 & 5727.028 & 1.080 & -0.798 & & & \\
\hline 23.00 & 5727.035 & 1.080 & -1.490 & & & \\
\hline 23.00 & 5727.040 & 1.080 & -2.605 & & & \\
\hline 23.00 & 5727.045 & 1.080 & -0.914 & & & \\
\hline 23.00 & 5727.051 & 1.080 & -1.417 & & & \\
\hline 23.00 & 5727.056 & 1.080 & -2.400 & & & \\
\hline 23.00 & 5727.060 & 1.080 & -1.043 & & & \\
\hline 23.00 & 5727.065 & 1.080 & -1.411 & & & \\
\hline 23.00 & 5727.069 & 1.080 & -2.303 & & & \\
\hline 23.00 & 5727.072 & 1.080 & -1.189 & & & \\
\hline 23.00 & 5727.075 & 1.080 & -1.458 & & & \\
\hline 23.00 & 5727.078 & 1.080 & -2.303 & & & \\
\hline 23.00 & 5727.081 & 1.080 & -1.359 & & & \\
\hline 23.00 & 5727.084 & 1.080 & -1.563 & & & \\
\hline 23.00 & 5727.086 & 1.080 & -2.458 & & & \\
\hline 23.00 & 5727.087 & 1.080 & -1.563 & & & \\
\hline 23.00 & 5727.089 & 1.080 & -1.759 & & & \\
\hline 23.00 & 5727.091 & 1.080 & -1.826 & & & \\
\hline 23.00 & 5727.619 & 1.050 & -1.456 & & & \\
\hline 23.00 & 5727.619 & 1.050 & -1.867 & & & \\
\hline 23.00 & 5727.653 & 1.050 & -1.753 & & & \\
\hline 23.00 & 5727.653 & 1.050 & -2.072 & & & \\
\hline 23.00 & 5727.654 & 1.050 & -1.867 & & & \\
\hline 23.00 & 5727.681 & 1.050 & -1.753 & & & \\
\hline 23.00 & 5727.681 & 1.050 & -1.878 & & & \\
\hline 23.00 & 5727.681 & 1.050 & -9.850 & & & \\
\hline 23.00 & 5727.701 & 1.050 & -2.054 & & & \\
\hline 23.00 & 5727.702 & 1.050 & -1.878 & & & \\
\hline 23.00 & 6039.726 & 1.063 & -0.650 & & & \\
\hline 23.00 & 6081.417 & 1.050 & -1.814 & & & \\
\hline 23.00 & 6081.418 & 1.050 & -1.638 & & & \\
\hline 23.00 & 6081.428 & 1.050 & -1.638 & & & \\
\hline 23.00 & 6081.428 & 1.050 & -9.610 & & & \\
\hline 23.00 & 6081.429 & 1.050 & -1.513 & & & \\
\hline 23.00 & 6081.443 & 1.050 & -1.513 & & & \\
\hline 23.00 & 6081.443 & 1.050 & -1.832 & & & \\
\hline 23.00 & 6081.444 & 1.050 & -1.627 & & & \\
\hline 23.00 & 6081.461 & 1.050 & -1.627 & & & \\
\hline 23.00 & 6081.462 & 1.050 & -1.216 & & & \\
\hline 23.00 & 6090.194 & 1.080 & -0.700 & & & \\
\hline 23.00 & 6090.201 & 1.080 & -0.841 & & & \\
\hline 23.00 & 6090.207 & 1.080 & -1.005 & & & \\
\hline 23.00 & 6090.208 & 1.080 & -1.540 & & & \\
\hline 23.00 & 6090.213 & 1.080 & -1.203 & & & \\
\hline 23.00 & 6090.213 & 1.080 & -1.344 & & & \\
\hline 23.00 & 6090.217 & 1.080 & -1.290 & & & \\
\hline 23.00 & 6090.217 & 1.080 & -1.458 & & & \\
\hline 23.00 & 6090.220 & 1.080 & -2.655 & & & \\
\hline 23.00 & 6090.221 & 1.080 & -1.312 & & & \\
\hline 23.00 & 6090.221 & 1.080 & -1.846 & & & \\
\hline 23.00 & 6090.223 & 1.080 & -1.403 & & & \\
\hline 23.00 & 6090.223 & 1.080 & -2.244 & & & \\
\hline 23.00 & 6090.225 & 1.080 & -1.591 & & & \\
\hline 23.00 & 6090.225 & 1.080 & -2.022 & & & \\
\hline 23.00 & 6090.226 & 1.080 & -1.897 & & & \\
\hline 23.00 & 6090.227 & 1.080 & -1.846 & & & \\
\hline 23.00 & 6090.227 & 1.080 & -1.876 & & & \\
\hline 23.00 & 6111.592 & 1.042 & -1.701 & & & \\
\hline 23.00 & 6111.632 & 1.042 & -1.224 & & & \\
\hline 23.00 & 6111.656 & 1.042 & -1.224 & & & \\
\hline 23.00 & 6111.696 & 1.042 & -1.370 & & & \\
\hline
\end{tabular}


Table 1. continued.

\begin{tabular}{|c|c|c|c|c|c|c|}
\hline Element & $\begin{array}{c}\lambda \\
{[\AA]}\end{array}$ & $\begin{array}{c}\mathrm{EP} \\
{[\mathrm{eV}]}\end{array}$ & $\begin{array}{r}\log g f \\
{[\operatorname{dex}]}\end{array}$ & $\begin{array}{l}E W_{1} \\
{[\mathrm{~m} \AA]}\end{array}$ & $\begin{array}{l}E W_{2} \\
{[\mathrm{~m} \AA]}\end{array}$ & $\begin{array}{c}E W_{\text {Sun }} \\
{[\mathrm{m} \AA]}\end{array}$ \\
\hline 23.00 & 6119.528 & 1.063 & -0.360 & & & \\
\hline 23.00 & 6199.149 & 0.286 & -2.133 & & & \\
\hline 23.00 & 6199.167 & 0.286 & -2.238 & & & \\
\hline 23.00 & 6199.182 & 0.286 & -2.354 & & & \\
\hline 23.00 & 6199.197 & 0.286 & -2.483 & & & \\
\hline 23.00 & 6199.201 & 0.286 & -3.141 & & & \\
\hline 23.00 & 6199.209 & 0.286 & -2.629 & & & \\
\hline 23.00 & 6199.212 & 0.286 & -2.930 & & & \\
\hline 23.00 & 6199.221 & 0.286 & -2.799 & & & \\
\hline 23.00 & 6199.221 & 0.286 & -2.857 & & & \\
\hline 23.00 & 6199.229 & 0.286 & -2.851 & & & \\
\hline 23.00 & 6199.230 & 0.286 & -3.003 & & & \\
\hline 23.00 & 6199.235 & 0.286 & -2.898 & & & \\
\hline 23.00 & 6199.238 & 0.286 & -3.266 & & & \\
\hline 23.00 & 6199.240 & 0.286 & -3.003 & & & \\
\hline 23.00 & 6199.243 & 0.286 & -3.199 & & & \\
\hline 23.00 & 6199.246 & 0.286 & -4.443 & & & \\
\hline 23.00 & 6199.251 & 0.286 & -4.045 & & & \\
\hline 23.00 & 6199.253 & 0.286 & -3.840 & & & \\
\hline 23.00 & 6199.253 & 0.286 & -3.898 & & & \\
\hline 23.00 & 6199.255 & 0.286 & -3.743 & & & \\
\hline 23.00 & 6199.255 & 0.286 & -3.743 & & & \\
\hline 23.00 & 6242.798 & 0.262 & -2.054 & & & \\
\hline 23.00 & 6242.798 & 0.262 & -2.521 & & & \\
\hline 23.00 & 6242.829 & 0.262 & -2.375 & & & \\
\hline 23.00 & 6242.837 & 0.262 & -2.375 & & & \\
\hline 23.00 & 6242.852 & 0.262 & -2.396 & & & \\
\hline 23.00 & 6242.868 & 0.262 & -2.852 & & & \\
\hline 23.00 & 6243.045 & 0.300 & -2.712 & & & \\
\hline 23.00 & 6243.060 & 0.300 & -2.497 & & & \\
\hline 23.00 & 6243.075 & 0.300 & -2.420 & & & \\
\hline 23.00 & 6243.087 & 0.300 & -1.649 & & & \\
\hline 23.00 & 6243.087 & 0.300 & -2.409 & & & \\
\hline 23.00 & 6243.097 & 0.300 & -1.785 & & & \\
\hline 23.00 & 6243.099 & 0.300 & -2.452 & & & \\
\hline 23.00 & 6243.106 & 0.300 & -1.933 & & & \\
\hline 23.00 & 6243.109 & 0.300 & -2.555 & & & \\
\hline 23.00 & 6243.114 & 0.300 & -2.092 & & & \\
\hline 23.00 & 6243.118 & 0.300 & -2.776 & & & \\
\hline 23.00 & 6243.120 & 0.300 & -2.261 & & & \\
\hline 23.00 & 6243.125 & 0.300 & -2.428 & & & \\
\hline 23.00 & 6243.129 & 0.300 & -2.566 & & & \\
\hline 23.00 & 6243.132 & 0.300 & -2.580 & & & \\
\hline 23.00 & 6243.140 & 0.300 & -2.712 & & & \\
\hline 23.00 & 6243.142 & 0.300 & -2.776 & & & \\
\hline 23.00 & 6243.143 & 0.300 & -2.497 & & & \\
\hline 23.00 & 6243.145 & 0.300 & -2.555 & & & \\
\hline 23.00 & 6243.146 & 0.300 & -2.420 & & & \\
\hline 23.00 & 6243.146 & 0.300 & -2.452 & & & \\
\hline 23.00 & 6243.147 & 0.300 & -2.409 & & & \\
\hline 23.00 & 6285.160 & 0.275 & -1.540 & & & \\
\hline 24.00 & 4708.017 & 3.170 & 0.090 & 71.2 & 72.8 & 54.6 \\
\hline 24.00 & 4767.860 & 3.560 & -0.600 & 32.0 & 33.5 & 16.3 \\
\hline 24.00 & 4789.340 & 2.540 & -0.350 & 86.2 & 86.9 & 64.8 \\
\hline 24.00 & 4801.047 & 3.120 & -0.130 & 68.7 & 70.5 & 47.9 \\
\hline 24.00 & 4936.335 & 3.110 & -0.250 & 65.7 & 68.5 & 44.2 \\
\hline 24.00 & 5214.140 & 3.370 & -0.740 & 32.1 & 33.5 & 16.1 \\
\hline 24.00 & 5238.964 & 2.710 & -1.270 & 34.0 & 36.8 & 14.9 \\
\hline 24.00 & 5247.566 & 0.960 & -1.590 & 104.6 & 107.7 & 81.4 \\
\hline 24.00 & 5272.007 & 3.450 & -0.420 & 43.7 & 44.5 & 24.0 \\
\hline 24.00 & 5287.200 & 3.440 & -0.870 & 24.5 & 26.8 & 11.0 \\
\hline 24.00 & 5628.621 & 3.420 & -0.760 & 31.5 & 32.3 & 13.8 \\
\hline 24.00 & 5783.080 & 3.320 & -0.430 & 52.0 & 55.5 & 32.2 \\
\hline 24.00 & 5783.870 & 3.320 & -0.290 & 71.7 & 75.2 & 44.1 \\
\hline 24.00 & 5787.930 & 3.322 & -0.080 & 68.3 & 70.5 & 45.7 \\
\hline
\end{tabular}


Table 1. continued.

\begin{tabular}{|c|c|c|c|c|c|c|}
\hline Element & $\begin{array}{c}\lambda \\
[\AA]]\end{array}$ & $\begin{array}{c}\mathrm{EP} \\
{[\mathrm{eV}]}\end{array}$ & $\begin{array}{r}\log g f \\
{[\operatorname{dex}]}\end{array}$ & $\begin{array}{c}E W_{1} \\
{[\mathrm{m \AA}]}\end{array}$ & $\begin{array}{c}E W_{2} \\
{[\mathrm{m \AA}]}\end{array}$ & $\begin{array}{c}E W_{\text {Sun }} \\
{[\mathrm{m} \AA]}\end{array}$ \\
\hline 24.00 & 6330.100 & 0.941 & -2.900 & 53.9 & 56.4 & 25.8 \\
\hline 24.00 & 6882.477 & 3.438 & -0.375 & 59.5 & 62.6 & 32.5 \\
\hline 24.10 & 5237.328 & 4.070 & -1.090 & 60.6 & 59.7 & 52.5 \\
\hline 25.00 & 4709.690 & 2.886 & -1.096 & & & \\
\hline 25.00 & 4709.698 & 2.886 & -2.088 & & & \\
\hline 25.00 & 4709.698 & 2.886 & -2.088 & & & \\
\hline 25.00 & 4709.705 & 2.886 & -1.267 & & & \\
\hline 25.00 & 4709.711 & 2.886 & -1.906 & & & \\
\hline 25.00 & 4709.711 & 2.886 & -1.906 & & & \\
\hline 25.00 & 4709.717 & 2.886 & -1.452 & & & \\
\hline 25.00 & 4709.722 & 2.886 & -1.875 & & & \\
\hline 25.00 & 4709.723 & 2.886 & -1.875 & & & \\
\hline 25.00 & 4709.728 & 2.886 & -1.644 & & & \\
\hline 25.00 & 4709.731 & 2.886 & -1.940 & & & \\
\hline 25.00 & 4709.731 & 2.886 & -1.940 & & & \\
\hline 25.00 & 4709.735 & 2.886 & -1.819 & & & \\
\hline 25.00 & 4709.737 & 2.886 & -2.138 & & & \\
\hline 25.00 & 4709.737 & 2.886 & -2.138 & & & \\
\hline 25.00 & 4709.740 & 2.886 & -1.883 & & & \\
\hline 25.00 & 4739.068 & 2.939 & -1.632 & & & \\
\hline 25.00 & 4739.069 & 2.939 & -1.155 & & & \\
\hline 25.00 & 4739.087 & 2.939 & -1.530 & & & \\
\hline 25.00 & 4739.088 & 2.939 & -1.704 & & & \\
\hline 25.00 & 4739.089 & 2.939 & -1.632 & & & \\
\hline 25.00 & 4739.101 & 2.939 & -1.662 & & & \\
\hline 25.00 & 4739.102 & 2.939 & -3.240 & & & \\
\hline 25.00 & 4739.103 & 2.939 & -1.530 & & & \\
\hline 25.00 & 4739.111 & 2.939 & -2.030 & & & \\
\hline 25.00 & 4739.112 & 2.939 & -1.662 & & & \\
\hline 25.00 & 5004.892 & 2.918 & -1.630 & & & \\
\hline 26.00 & 4745.800 & 3.650 & -1.270 & 97.2 & 99.1 & 77.3 \\
\hline 26.00 & 4749.950 & 4.560 & -1.240 & 55.4 & 56.6 & 35.9 \\
\hline 26.00 & 4799.410 & 3.640 & -2.130 & 52.8 & 53.5 & 33.4 \\
\hline 26.00 & 4808.150 & 3.250 & -2.690 & 43.2 & 44.3 & 26.0 \\
\hline 26.00 & 4973.090 & 3.960 & -0.770 & 103.6 & 108.2 & 82.6 \\
\hline 26.00 & 5044.211 & 2.850 & -2.060 & 93.7 & 95.6 & 73.0 \\
\hline 26.00 & 5054.642 & 3.640 & -1.920 & 61.8 & 62.2 & 40.3 \\
\hline 26.00 & 5067.140 & 4.220 & -0.860 & 93.2 & 94.7 & 67.8 \\
\hline 26.00 & 5127.679 & 0.050 & -6.120 & 39.4 & 42.0 & 16.9 \\
\hline 26.00 & 5187.910 & 4.140 & -1.260 & 80.5 & 82.2 & 58.3 \\
\hline 26.00 & 5225.525 & 0.110 & -4.790 & 95.5 & 98.0 & 71.8 \\
\hline 26.00 & 5250.208 & 0.120 & -4.940 & 85.8 & 88.1 & 64.6 \\
\hline 26.00 & 5253.460 & 3.280 & -1.570 & 101.2 & 101.8 & 79.2 \\
\hline 26.00 & 5409.130 & 4.370 & -1.060 & 77.0 & 76.8 & 57.7 \\
\hline 26.00 & 5466.987 & 3.570 & -2.230 & 54.7 & 54.9 & 32.7 \\
\hline 26.00 & 5577.020 & 5.030 & -1.460 & 20.8 & 22.1 & 10.4 \\
\hline 26.00 & 5636.696 & 3.640 & -2.560 & 35.0 & 35.7 & 18.8 \\
\hline 26.00 & 5638.262 & 4.220 & -0.770 & 101.6 & 101.1 & 76.1 \\
\hline 26.00 & 5649.987 & 5.100 & -0.800 & 54.3 & 54.6 & 34.8 \\
\hline 26.00 & 5651.469 & 4.470 & -1.750 & 34.1 & 34.0 & 18.2 \\
\hline 26.00 & 5661.348 & 4.280 & -1.760 & 41.5 & 42.7 & 22.5 \\
\hline 26.00 & 5679.023 & 4.650 & -0.750 & 76.6 & 78.9 & 57.9 \\
\hline 26.00 & 5696.089 & 4.550 & -1.780 & 26.7 & 27.1 & 13.0 \\
\hline 26.00 & 5705.464 & 4.300 & -1.360 & 56.4 & 55.8 & 36.8 \\
\hline 26.00 & 5731.760 & 4.260 & -1.200 & 76.8 & 78.4 & 57.4 \\
\hline 26.00 & 5778.453 & 2.590 & -3.440 & 43.2 & 44.5 & 21.8 \\
\hline 26.00 & 5784.660 & 3.400 & -2.530 & 46.2 & 48.6 & 25.5 \\
\hline 26.00 & 5793.914 & 4.220 & -1.620 & 51.3 & 53.4 & 32.1 \\
\hline 26.00 & 5806.730 & 4.610 & -0.950 & 76.8 & 79.1 & 52.9 \\
\hline 26.00 & 5852.220 & 4.550 & -1.230 & 62.3 & 62.8 & 39.5 \\
\hline 26.00 & 5855.076 & 4.610 & -1.480 & 39.3 & 40.3 & 22.5 \\
\hline 26.00 & 5856.090 & 4.290 & -1.460 & 51.8 & 52.5 & 32.9 \\
\hline 26.00 & 5927.789 & 4.650 & -1.040 & 60.1 & 59.9 & 41.6 \\
\hline 26.00 & 5934.655 & 3.930 & -1.070 & 99.9 & 101.2 & 75.9 \\
\hline 26.00 & 6056.005 & 4.730 & -0.400 & 92.2 & 93.9 & 71.4 \\
\hline
\end{tabular}


Table 1. continued.

\begin{tabular}{|c|c|c|c|c|c|c|}
\hline Element & $\begin{array}{c}\lambda \\
{[\AA]} \\
{[\AA}\end{array}$ & $\begin{array}{c}\mathrm{EP} \\
{[\mathrm{eV}]}\end{array}$ & $\begin{array}{r}\log g f \\
{[\mathrm{dex}]}\end{array}$ & $\begin{array}{c}E W_{1} \\
{[\mathrm{~m} \AA]}\end{array}$ & $\begin{array}{l}E W_{2} \\
{[\mathrm{~m} \AA]}\end{array}$ & $\begin{array}{c}E W_{\text {Sun }} \\
{[\mathrm{m} \AA]}\end{array}$ \\
\hline 26.00 & 6079.009 & 4.650 & -1.020 & 64.7 & 63.9 & 44.7 \\
\hline 26.00 & 6082.711 & 2.220 & -3.570 & 55.0 & 57.6 & 33.8 \\
\hline 26.00 & 6093.644 & 4.610 & -1.300 & 49.1 & 48.5 & 29.6 \\
\hline 26.00 & 6127.910 & 4.140 & -1.400 & 69.5 & 68.3 & 49.6 \\
\hline 26.00 & 6151.618 & 2.180 & -3.280 & 70.3 & 71.5 & 49.0 \\
\hline 26.00 & 6157.728 & 4.080 & -1.220 & 79.9 & 81.3 & 60.8 \\
\hline 26.00 & 6165.360 & 4.140 & -1.460 & 61.7 & 63.7 & 43.1 \\
\hline 26.00 & 6213.430 & 2.220 & -2.520 & 102.8 & 106.8 & 81.3 \\
\hline 26.00 & 6219.281 & 2.200 & -2.430 & 116.5 & 118.3 & 88.7 \\
\hline 26.00 & 6226.736 & 3.880 & -2.100 & 49.4 & 49.9 & 28.5 \\
\hline 26.00 & 6252.555 & 2.400 & -1.690 & 153.3 & 157.8 & 118.9 \\
\hline 26.00 & 6270.225 & 2.860 & -2.540 & 73.7 & 75.2 & 51.2 \\
\hline 26.00 & 6271.279 & 3.330 & -2.700 & 41.3 & 41.8 & 22.9 \\
\hline 26.00 & 6335.330 & 2.200 & -2.260 & 121.7 & 123.9 & 95.9 \\
\hline 26.00 & 6392.539 & 2.280 & -4.030 & 35.2 & 36.4 & 15.8 \\
\hline 26.00 & 6481.870 & 2.280 & -2.980 & 85.7 & 87.0 & 63.6 \\
\hline 26.00 & 6518.370 & 2.830 & -2.450 & 76.7 & 78.6 & 56.2 \\
\hline 26.00 & 6593.871 & 2.430 & -2.390 & 109.4 & 110.2 & 82.7 \\
\hline 26.00 & 6597.561 & 4.800 & -0.970 & 62.3 & 63.1 & 43.2 \\
\hline 26.00 & 6625.022 & 1.010 & -5.340 & 34.7 & 36.6 & 14.8 \\
\hline 26.00 & 6699.142 & 4.593 & -2.101 & 18.4 & 18.8 & 8.1 \\
\hline 26.00 & 6703.567 & 2.760 & -3.020 & 60.7 & 61.7 & 36.8 \\
\hline 26.00 & 6705.102 & 4.610 & -0.980 & 69.2 & 70.3 & 46.0 \\
\hline 26.00 & 6713.745 & 4.800 & -1.400 & 37.7 & 37.9 & 20.5 \\
\hline 26.00 & 6725.357 & 4.100 & -2.190 & 33.2 & 34.6 & 16.5 \\
\hline 26.00 & 6726.667 & 4.610 & -1.030 & 66.0 & 66.4 & 46.5 \\
\hline 26.00 & 6733.151 & 4.640 & -1.470 & 45.9 & 47.2 & 26.1 \\
\hline 26.00 & 6750.152 & 2.420 & -2.620 & 98.2 & 100.9 & 73.9 \\
\hline 26.00 & 6806.845 & 2.730 & -3.110 & 58.1 & 60.1 & 34.2 \\
\hline 26.00 & 6810.263 & 4.610 & -0.990 & 70.3 & 70.5 & 49.5 \\
\hline 26.00 & 6828.590 & 4.640 & -0.820 & 77.2 & 77.8 & 54.3 \\
\hline 26.00 & 6837.006 & 4.590 & -1.690 & 33.3 & 34.1 & 17.9 \\
\hline 26.00 & 6842.690 & 4.640 & -1.220 & 58.3 & 58.0 & 36.7 \\
\hline 26.00 & 6843.656 & 4.550 & -0.830 & 83.8 & 83.8 & 60.4 \\
\hline 26.00 & 6858.150 & 4.610 & -0.940 & 69.9 & 70.9 & 50.9 \\
\hline 26.00 & 6999.880 & 4.100 & -1.460 & 75.1 & 75.5 & 54.0 \\
\hline 26.00 & 7132.990 & 4.080 & -1.650 & 63.0 & 63.1 & 41.8 \\
\hline 26.00 & 7401.685 & 4.186 & -1.500 & 60.0 & 59.9 & 40.5 \\
\hline 26.00 & 7418.670 & 4.140 & -1.380 & 71.2 & 71.5 & 47.5 \\
\hline 26.10 & 4620.510 & 2.830 & -3.210 & 60.0 & 57.9 & 52.4 \\
\hline 26.10 & 4993.340 & 2.810 & -3.730 & 44.3 & 43.4 & 38.3 \\
\hline 26.10 & 5414.073 & 3.220 & -3.580 & 34.3 & 32.8 & 26.6 \\
\hline 26.10 & 6084.111 & 3.200 & -3.830 & 25.9 & 24.8 & 20.1 \\
\hline 26.10 & 6416.919 & 3.890 & -2.750 & 47.2 & 45.4 & 38.5 \\
\hline 26.10 & 6432.680 & 2.890 & -3.570 & 46.7 & 45.7 & 39.5 \\
\hline 26.10 & 6456.383 & 3.900 & -2.050 & 67.4 & 63.6 & 61.7 \\
\hline 26.10 & 7711.721 & 3.903 & -2.500 & 53.1 & 50.2 & 44.5 \\
\hline 27.00 & 4792.846 & 3.250 & -0.070 & & & \\
\hline 27.00 & 4813.467 & 3.213 & 0.050 & & & \\
\hline 27.00 & 5212.691 & 3.512 & -0.110 & & & \\
\hline 27.00 & 5247.911 & 1.784 & -2.070 & & & \\
\hline 27.00 & 5647.234 & 2.278 & -1.560 & & & \\
\hline 27.00 & 6093.143 & 1.739 & -2.440 & & & \\
\hline 27.00 & 6454.990 & 3.629 & -0.250 & & & \\
\hline 28.00 & 4831.180 & 3.610 & -0.320 & 91.9 & 92.4 & 73.2 \\
\hline 28.00 & 4866.270 & 3.540 & -0.210 & 102.4 & 101.3 & 77.1 \\
\hline 28.00 & 4913.980 & 3.740 & -0.660 & 75.2 & 75.2 & 55.7 \\
\hline 28.00 & 4946.040 & 3.800 & -1.220 & 51.2 & 53.0 & 28.0 \\
\hline 28.00 & 4952.290 & 3.610 & -1.260 & 54.5 & 55.7 & 32.4 \\
\hline 28.00 & 4953.208 & 3.740 & -0.660 & 75.6 & 76.2 & 56.0 \\
\hline 28.00 & 4976.135 & 3.610 & -1.250 & 51.3 & 49.7 & 29.0 \\
\hline 28.00 & 5010.938 & 3.640 & -0.870 & 65.0 & 65.6 & 48.4 \\
\hline 28.00 & 5082.350 & 3.660 & -0.590 & 99.3 & 96.8 & 67.0 \\
\hline 28.00 & 5084.110 & 3.680 & -0.060 & 108.3 & 109.8 & 88.1 \\
\hline 28.00 & 5094.420 & 3.830 & -1.070 & 47.7 & 47.3 & 28.7 \\
\hline
\end{tabular}


Table 1. continued.

\begin{tabular}{|c|c|c|c|c|c|c|}
\hline Element & $\begin{array}{c}\lambda \\
[\AA]]\end{array}$ & $\begin{array}{c}\mathrm{EP} \\
{[\mathrm{eV}]}\end{array}$ & $\begin{array}{r}\log g f \\
{[\operatorname{dex}]}\end{array}$ & $\begin{array}{c}E W_{1} \\
{[\mathrm{m \AA}]}\end{array}$ & $\begin{array}{c}E W_{2} \\
{[\mathrm{~m} \AA]}\end{array}$ & $\begin{array}{c}E W_{\text {Sun }} \\
{[\mathrm{m} \AA]}\end{array}$ \\
\hline 28.00 & 5157.980 & 3.610 & -1.510 & 34.4 & 35.2 & 16.9 \\
\hline 28.00 & 5578.729 & 1.680 & -2.570 & 80.5 & 83.1 & 57.4 \\
\hline 28.00 & 5589.358 & 3.900 & -1.140 & 45.2 & 45.7 & 28.1 \\
\hline 28.00 & 5593.746 & 3.900 & -0.780 & 64.7 & 66.0 & 43.8 \\
\hline 28.00 & 5625.320 & 4.090 & -0.730 & 58.4 & 56.6 & 37.1 \\
\hline 28.00 & 5628.350 & 4.090 & -1.320 & 32.1 & 30.8 & 14.3 \\
\hline 28.00 & 5638.750 & 3.900 & -1.700 & 22.7 & 22.2 & 9.6 \\
\hline 28.00 & 5641.880 & 4.110 & -1.020 & 44.8 & 44.9 & 23.2 \\
\hline 28.00 & 5643.078 & 4.160 & -1.250 & 29.9 & 30.0 & 14.6 \\
\hline 28.00 & 5694.990 & 4.090 & -0.630 & 62.0 & 62.7 & 42.7 \\
\hline 28.00 & 5748.360 & 1.680 & -3.240 & 51.1 & 52.2 & 27.5 \\
\hline 28.00 & 5754.670 & 1.930 & -1.850 & 99.5 & 101.9 & 78.7 \\
\hline 28.00 & 5805.217 & 4.170 & -0.640 & 61.6 & 63.6 & 40.4 \\
\hline 28.00 & 5847.010 & 1.676 & -3.410 & 46.2 & 48.0 & 22.6 \\
\hline 28.00 & 6086.282 & 4.270 & -0.510 & 67.1 & 68.6 & 43.4 \\
\hline 28.00 & 6111.080 & 4.088 & -0.810 & 58.5 & 58.4 & 33.3 \\
\hline 28.00 & 6119.760 & 4.270 & -1.316 & 23.5 & 24.2 & 10.6 \\
\hline 28.00 & 6128.984 & 1.677 & -3.360 & 50.1 & 49.0 & 25.0 \\
\hline 28.00 & 6130.135 & 4.270 & -0.960 & 41.4 & 40.5 & 21.9 \\
\hline 28.00 & 6175.370 & 4.089 & -0.550 & 69.1 & 69.2 & 49.0 \\
\hline 28.00 & 6176.811 & 4.090 & -0.260 & 87.5 & 87.9 & 62.9 \\
\hline 28.00 & 6177.242 & 1.830 & -3.510 & 34.2 & 33.7 & 13.5 \\
\hline 28.00 & 6186.717 & 4.110 & -0.960 & 52.0 & 52.6 & 30.6 \\
\hline 28.00 & 6204.604 & 4.090 & -1.140 & 42.2 & 43.1 & 21.7 \\
\hline 28.00 & 6223.971 & 4.105 & -1.466 & 47.8 & 48.8 & 27.0 \\
\hline 28.00 & 6230.100 & 4.110 & -1.132 & 40.3 & 42.4 & 19.3 \\
\hline 28.00 & 6322.169 & 4.154 & -1.210 & 36.6 & 36.6 & 18.5 \\
\hline 28.00 & 6360.810 & 4.170 & -1.150 & 37.0 & 35.1 & 16.6 \\
\hline 28.00 & 6378.233 & 4.154 & -1.386 & 55.0 & 55.4 & 31.0 \\
\hline 28.00 & 6598.611 & 4.236 & -0.910 & 43.2 & 43.9 & 24.8 \\
\hline 28.00 & 6635.130 & 4.420 & -0.720 & 44.8 & 44.3 & 23.3 \\
\hline 28.00 & 6643.630 & 1.680 & -2.000 & 126.8 & 126.5 & 92.1 \\
\hline 28.00 & 6767.772 & 1.830 & -2.170 & 103.4 & 106.2 & 78.4 \\
\hline 28.00 & 6772.315 & 3.660 & -0.990 & 72.6 & 73.9 & 49.2 \\
\hline 28.00 & 6842.043 & 3.658 & -1.500 & 45.0 & 40.8 & 24.2 \\
\hline 28.00 & 7715.591 & 3.700 & -1.010 & 76.3 & 77.0 & 50.0 \\
\hline 28.00 & 7727.624 & 3.680 & -0.400 & 117.5 & 118.2 & 91.0 \\
\hline 28.00 & 7748.890 & 3.700 & -0.380 & 115.6 & 116.4 & 85.0 \\
\hline 28.00 & 7797.586 & 3.890 & -0.340 & 104.0 & 107.2 & 74.0 \\
\hline 29.00 & 5218.197 & 3.814 & 0.480 & & & \\
\hline 29.00 & 7933.096 & 3.783 & -0.877 & & & \\
\hline 29.00 & 7933.098 & 3.783 & -0.877 & & & \\
\hline 29.00 & 7933.119 & 3.783 & -0.877 & & & \\
\hline 29.00 & 7933.119 & 3.783 & -0.877 & & & \\
\hline 29.00 & 7933.134 & 3.783 & -1.576 & & & \\
\hline 29.00 & 7933.135 & 3.783 & -1.576 & & & \\
\hline 29.00 & 7933.155 & 3.783 & -0.877 & & & \\
\hline 29.00 & 7933.157 & 3.783 & -0.877 & & & \\
\hline 38.00 & 4607.338 & 0.000 & 0.283 & 68.5 & 69.2 & 46.9 \\
\hline 39.10 & 4854.867 & 0.992 & -0.380 & 55.7 & 55.1 & 47.7 \\
\hline 39.10 & 4883.685 & 1.084 & 0.070 & 62.3 & 62.7 & 56.8 \\
\hline 39.10 & 4900.110 & 1.033 & -0.090 & 61.7 & 60.1 & 54.1 \\
\hline 39.10 & 5087.420 & 1.084 & -0.170 & 58.3 & 57.9 & 47.0 \\
\hline 39.10 & 5200.413 & 0.992 & -0.570 & 47.8 & 47.2 & 37.9 \\
\hline 56.00 & 5853.686 & 0.604 & -2.066 & & & \\
\hline 56.00 & 5853.687 & 0.604 & -2.066 & & & \\
\hline 56.00 & 5853.687 & 0.604 & -2.009 & & & \\
\hline 56.00 & 5853.688 & 0.604 & -2.009 & & & \\
\hline 56.00 & 5853.689 & 0.604 & -2.215 & & & \\
\hline 56.00 & 5853.689 & 0.604 & -2.215 & & & \\
\hline 56.00 & 5853.690 & 0.604 & -1.010 & & & \\
\hline 56.00 & 5853.690 & 0.604 & -1.466 & & & \\
\hline 56.00 & 5853.690 & 0.604 & -1.914 & & & \\
\hline 56.00 & 5853.690 & 0.604 & -2.620 & & & \\
\hline
\end{tabular}


Table 1. continued.

\begin{tabular}{|c|c|c|c|c|c|c|}
\hline Element & $\begin{array}{c}\lambda \\
{[\AA]}\end{array}$ & $\begin{array}{c}\text { EP } \\
{[\mathrm{eV}]}\end{array}$ & $\begin{array}{r}\log g f \\
{[\operatorname{dex}]}\end{array}$ & $\begin{array}{l}E W_{1} \\
{[\mathrm{~m} \AA]}\end{array}$ & $\begin{array}{c}E W_{2} \\
{[\mathrm{~m} \AA]}\end{array}$ & $\begin{array}{c}E W_{\text {Sun }} \\
{[\mathrm{m} \AA]}\end{array}$ \\
\hline 56.00 & 5853.690 & 0.604 & -1.010 & & & \\
\hline 56.00 & 5853.690 & 0.604 & -1.466 & & & \\
\hline 56.00 & 5853.690 & 0.604 & -1.914 & & & \\
\hline 56.00 & 5853.690 & 0.604 & -2.620 & & & \\
\hline 56.00 & 5853.690 & 0.604 & -1.010 & & & \\
\hline 56.00 & 5853.691 & 0.604 & -2.215 & & & \\
\hline 56.00 & 5853.692 & 0.604 & -2.215 & & & \\
\hline 56.00 & 5853.693 & 0.604 & -2.009 & & & \\
\hline 56.00 & 5853.693 & 0.604 & -2.009 & & & \\
\hline 56.00 & 5853.694 & 0.604 & -2.066 & & & \\
\hline 56.00 & 5853.694 & 0.604 & -2.066 & & & \\
\hline 56.00 & 6141.725 & 0.704 & -2.456 & & & \\
\hline 56.00 & 6141.725 & 0.704 & -2.456 & & & \\
\hline 56.00 & 6141.727 & 0.704 & -1.311 & & & \\
\hline 56.00 & 6141.727 & 0.704 & -1.311 & & & \\
\hline 56.00 & 6141.728 & 0.704 & -2.284 & & & \\
\hline 56.00 & 6141.728 & 0.704 & -2.284 & & & \\
\hline 56.00 & 6141.729 & 0.704 & -0.503 & & & \\
\hline 56.00 & 6141.729 & 0.704 & -1.214 & & & \\
\hline 56.00 & 6141.729 & 0.704 & -0.503 & & & \\
\hline 56.00 & 6141.729 & 0.704 & -1.214 & & & \\
\hline 56.00 & 6141.730 & 0.704 & -0.077 & & & \\
\hline 56.00 & 6141.730 & 0.704 & -0.077 & & & \\
\hline 56.00 & 6141.730 & 0.704 & -0.077 & & & \\
\hline 56.00 & 6141.731 & 0.704 & -0.709 & & & \\
\hline 56.00 & 6141.731 & 0.704 & -1.327 & & & \\
\hline 56.00 & 6141.731 & 0.704 & -0.709 & & & \\
\hline 56.00 & 6141.731 & 0.704 & -1.327 & & & \\
\hline 56.00 & 6141.732 & 0.704 & -0.959 & & & \\
\hline 56.00 & 6141.732 & 0.704 & -1.281 & & & \\
\hline 56.00 & 6141.732 & 0.704 & -0.959 & & & \\
\hline 56.00 & 6141.733 & 0.704 & -1.281 & & & \\
\hline 56.00 & 6496.898 & 0.604 & -1.886 & & & \\
\hline 56.00 & 6496.899 & 0.604 & -1.886 & & & \\
\hline 56.00 & 6496.901 & 0.604 & -1.186 & & & \\
\hline 56.00 & 6496.902 & 0.604 & -1.186 & & & \\
\hline 56.00 & 6496.906 & 0.604 & -0.739 & & & \\
\hline 56.00 & 6496.906 & 0.604 & -0.739 & & & \\
\hline 56.00 & 6496.910 & 0.604 & -0.380 & & & \\
\hline 56.00 & 6496.910 & 0.604 & -0.380 & & & \\
\hline 56.00 & 6496.910 & 0.604 & -0.380 & & & \\
\hline 56.00 & 6496.916 & 0.604 & -1.583 & & & \\
\hline 56.00 & 6496.916 & 0.604 & -1.583 & & & \\
\hline 56.00 & 6496.917 & 0.604 & -1.186 & & & \\
\hline 56.00 & 6496.918 & 0.604 & -1.186 & & & \\
\hline 56.00 & 6496.920 & 0.604 & -1.186 & & & \\
\hline 56.00 & 6496.922 & 0.604 & -1.186 & & & \\
\hline
\end{tabular}

